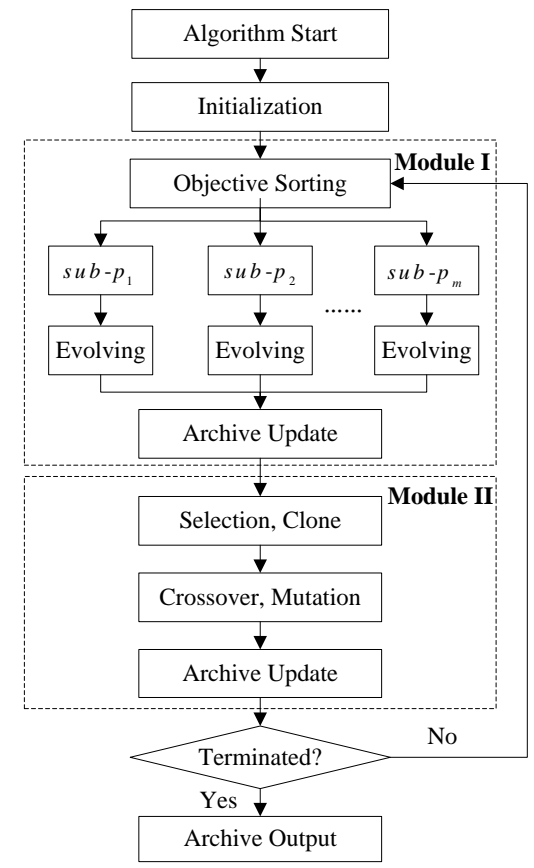

Figure 1 . The framework of the proposed algorithm with two evolutionary modules 


\title{
A Double-module Immune Algorithm for Multi-objective Optimization Problems
}

Zhengping Liang, Ruizhen Song, Qiuzhen Lin*, Zhihua Du, Jianyong Chen, Zhong Ming, Jianping Yu College of Computer Science and Software Engineering, Shenzhen University, Shenzhen, P.R. China,

\begin{abstract}
Multi-objective optimization problems (MOPs) have become a research hotspot, as they are commonly encountered in scientific and engineering applications. When solving some complex MOPs, it is quite difficult to locate the entire Pareto-optimal front. To better settle this problem, a novel double-module immune algorithm named DMMO is presented, where two evolutionary modules are embedded to simultaneously improve the convergence speed and population diversity. The first module is designed to optimize each objective independently by using a sub-population composed with the competitive individuals in this objective. Differential evolution crossover is performed here to enhance the corresponding objective. The second one follows the traditional procedures of immune algorithm, where proportional cloning, recombination and hyper-mutation operators are operated to concurrently strengthen the multiple objectives. The performance of DMMO is validated by 16 benchmark problems, and further compared with several multi-objective algorithms, such as NSGA-II, SPEA2, SMSEMOA, MOEA/D, SMPSO, NNIA and MIMO. Experimental studies indicate that DMMO performs better than the compared targets on most of test problems and the advantages of double modules in DMMO are also analyzed.
\end{abstract}

Keywords: Multi-objective optimization; Double-module framework; Immune algorithm; Differential evolution;

\section{Introduction}

Over the last decades, multi-objective optimization problems (MOPs) have attracted a great interest of researchers, which are motivated by the real-world engineering problems, such as job shop scheduling [1-2], water distribution network design [3-4], antenna design [5] and power supply management [6]. For example, the objectives of makespan, total workload, and critical workload in job shop scheduling are all required to be minimized, while the network cost and total head loss in pipes are preferred to be optimized in the design of water distribution network. Obviously, unlike single-objective optimization problems that only seek for one optimal value, MOPs bring new challenges as they need to simultaneously optimize several conflicting objectives. Thus, the output of 
MOPs is generally a number of equally-optimal solutions termed Pareto-optimal solutions when considering all the objectives. All Pareto-optimal solutions compose the Pareto-optimal set (PS), whose projection in the objective space is termed Pareto-optimal front (PF). Thus, the aim of MOPs is to achieve a subset of PS that is distributed uniformly along the true PF [7-8], which can be provided to the decision maker as the available choices for various practical cases.

Traditionally, multiple objectives are simply aggregated into a single objective optimization problem and then several runs of optimization algorithm are executed in order to find a set of optimal solutions [9]. The representative algorithms include the weighting method, the constraint approach, goal programming and the minmax formulation [10-11]. However, it is pretty difficult to achieve a satisfactory result in limited time using these traditional approaches as it needs to explore a huge solution space in MOPs by multiple runs for gathering a set of optimal solutions. Thus, evolutionary algorithms (EAs) are presented for solving MOPs, which have been demonstrated to be an effective method for MOPs as the population-based searching nature of EAs can obtain multiple Pareto-optimal solutions in a single run [7-8]. Since the first multi-objective EA (MOEA) named vector evaluated genetic algorithm (VEGA) was proposed by Schaffer [12], various MOEAs were developed afterward [7-8, 13-16], among which a fast non-dominated sorting genetic algorithm (NSGA-II) [7] and an improved strength Pareto EA (SPEA2) [8] are acknowledged as the two state-of-the-art MOEAs. A fast non-dominated sorting approach and elitism strategy are proposed in NSGA-II, while a new fitness assignment strategy and an enhanced archive truncation technique are presented in SPEA2. To further enhance the performance, various promising approaches are designed afterward, such as IBEA [17] and SMSEMOA [18] that embed the quality indicators into the selection procedure, ParEGO [19] that builds a Gaussian processes model of the search landscape to solve expensive MOPs, and MO-CMA-ES [20] that extends the covariance matrix adaptation evolution strategy (CMA-ES) to tackle MOPs. More recently, many competitive MOEAs have been reported with better performance [21-29]. For example, a neighborhood knowledge-based EA for MOPs (NKBA) [21] exploits the neighborhood knowledge systematically for acquiring more thorough local search. A diversity maintain strategy [22] is designed for MOPs to compute a solution density estimation of the archive through a binary space partitioning tree. A novel adaptive local search approach is embedded into MOEAs [23] for accelerating the convergence speed. With more and more MOEAs proposed, the performance for solving MOPs is constantly improved.

On the other hand, other nature-inspired algorithms such as particle swarm optimization (PSO) [30-31], ant colony algorithm [32-33] and artificial immune algorithm [34-35] are also investigated to solve MOPs. Among them, artificial immune algorithm is designed by mimicking the information processing procedures of artificial immune system (AIS), which has attracted much attention and been successfully applied in many research fields, such as data mining, computer security, 
optimization, and fault diagnosis [36-37]. Especially for tackling MOPs, artificial immune algorithm has demonstrated to be very competitive as experimentally studied in [38-41]. A more detail introduction of multi-objective immune algorithms is presented in Section 2.3.

It is recently found that single-objective optimization process can be effectively embedded into multi-objective algorithms for enhancing their performance. For example, MOEA/D decomposes MOPs into a set of single-objective aggregation problems based on the predefined weight vectors and then assigns each individual to optimize the corresponding subproblem [27]; CMPSO utilizes PSO to optimize each objective using one sub-population and then employs the shared external archive for information exchange between all the objectives [30]; the membrane computing algorithm proposed in [42-43] use several cells to optimize each objective and then employs other cells to enhance all the objectives simultaneously. Their promising performance motivates us to study the possibility of embedding single-objective optimization process into artificial immune algorithm, which may further enhance the performance of our previous algorithms [39-40]. Therefore, a double-module immune algorithm for MOPs (DMMO) is accordingly designed, which integrates a single-objective optimization module into the traditional immune algorithm and makes two evolutionary modules cooperatively evolved to solve MOPs. Although the idea to embed single-objective optimization process for MOPs has been investigated in MOEA/D [27], CMPSO [30] and the membrane computing algorithm [42-43], our framework with two evolutionary modules makes DMMO totally different from MOEA/D and CMPSO as they only adopt a single evolutionary module to evolve the population. For the membrane computing algorithm in [42-43], DMMO is essentially different as they are designed based on different evolutionary computing frameworks with various evolutionary operators. Our first module is aimed at improving each objective independently while the second one follows the traditional immune algorithm to strengthen the multiple objectives simultaneously. The cooperation of two modules is expected to accelerate the convergence speed and maintain the population diversity, which is validated by the experimental studies. When compared with various multi-objective algorithms, e.g., NSGA-II [7], SPEA2 [8], SMSEMOA [18], MOEA/D [27], SMPSO [31], NNIA [38] and MIMO [40], the experimental results show that DMMO performs better on most of test problems.

The rest of this paper is organized as follows. Section 2 introduces the related background, such as MOPs, AIS and some relevant immune algorithms. In Section 3, the proposed DMMO algorithm is described in detail. Section 4 gives the experimental comparisons of DMMO with other algorithms, which validate the superior performance of DMMO. Moreover, the advantages of double modules in DMMO are also experimentally analyzed. At last, further discussions of the double-module framework are given in Section 5 and conclusions are drawn in Section 6.

\section{Background}




\subsection{Multi-objective Optimization Problems}

Multi-objective optimization problems for minimization can be mathematically described by

$$
\text { Minimize } f(x)=\left\{f_{1}(x), f_{2}(x), \ldots, f_{m}(x)\right\}
$$

where $x$ is a decision vector as represented by $x=\left\{x_{1}, x_{2}, \ldots, x_{n}\right\} \in \Omega, n$ and $m$ are respectively the dimensions of the decision and objective vectors, and $\Omega$ is the feasible region in decision space. To distinguish the superiority of each solution, the definitions of Pareto optimality are important, which are described as follows [44].

Definition 1: Pareto domination. A decision vector $x_{A} \in \Omega$ is said to dominate another decision vector $x_{B} \in \Omega$ (noted as $x_{A} \succ x_{B}$ ) if and only if the following conditions are true.

$$
\left(\forall i \in\{1,2, \ldots, m\}: f_{i}\left(x_{A}\right) \leq f_{i}\left(x_{B}\right)\right) \wedge\left(\exists j \in\{1,2, \ldots, m\}: f_{j}\left(x_{A}\right)<f_{j}\left(x_{B}\right)\right)
$$

Definition 2: Pareto optimal. A decision vector $x^{*} \in \Omega$ is called a Pareto-optimal or non-dominated solution if and only if there does not exist another decision vector $x \in \Omega$ that can dominate $x^{*}$, which can be described as follows.

$$
\neg \exists x \succ x^{*}, x \in \Omega
$$

Definition 3: Pareto-optimal Set (PS). All Pareto-optimal vectors compose a PS, which can be defined by

$$
P S=\{x \mid \neg \exists y \in \Omega: y \succ x\}
$$

Definition4: Pareto-optimal Front (PF). The corresponding map of PS on the objective space is called PF, which is represented by

$$
P F=\left\{f(x)=\left\{f_{1}(x), f_{2}(x), \ldots, f_{m}(x)\right\} \mid x \in P S\right\}
$$

\subsection{Artificial Immune System}

Artificial immune system is an interesting bio-inspired intelligent approach that simulates the information processing procedures of biologic immune system [45-46]. When foreign antigens are detected in biologic immune system, its B-cell is adapted correspondingly to eliminate the intruders, which is realized by the processes known as clonal selection and affinity maturation through hyper-mutation. Antibodies that can better recognize an antigen will be selected to proliferate by cloning, which is known as the process of clonal selection. Then, hyper-mutation implements an affinity maturation process proportional to the fitness values in order to generate the matured population. At last, some antibodies with better affinities will be remained as memory cells to prevent the re-intrusion of the previous antigens. This information processing principle gives some inspirations to design artificial immune algorithm, which improves the convergence speed and maintain the diversity of the antibody population.

In multi-objective immune algorithm, the problems and the corresponding constraints can be treated as the antigen while a potential solution can be seen as an antibody. That is to say, as the 
MOPs defined in Eq. (1), a solution vector $x=\left\{x_{1}, x_{2}, \ldots, x_{n}\right\} \in \Omega$ is considered as an antibody and $f(x)=\left\{f_{1}(x), f_{2}(x), \ldots, f_{m}(x)\right\}$ is regarded as an antigen. An antibody population is made up by a set of antibodies. When an antibody is called a non-dominated antibody, it indicates that there does not exist another antibody in the antibody population that can dominate it.

\subsection{Related Work on Immune Algorithm}

To solve optimization problems, most of immune algorithms are designed by mimicking the two important immune principles, such as clonal selection and affinity maturation by hyper-mutation. The first immune optimization algorithm [47] was developed for solving single-objective optimization problem with an abstract clonal selection concept. Then, De Castro and Timmis proposed an artificial immune network algorithm named opt-aiNet [45] for multimodal optimization, in which the immune network decides which antibody will be cloned, repressed or maintained. After that, the learning and optimization algorithm using the clonal selection principle (CLONALG) [46], which takes the affinity maturation of immune response into account, was adapted to solve multimodal and combinatorial optimization problems.

Motivated by the promising performance of AIS in single-objective optimization problems, immune algorithms are extended to tackle MOPs. The multi-objective immune system algorithm (MISA) [34], as an early multi-objective immune algorithm, was presented based on clonal selection principle, in which an external memory is used to preserve the non-dominated antibodies and only the highest fitness antibodies will be selected to proliferate. In [48, 49], a vector artificial immune system (VAIS) was reported, which is extended from opt-aiNet [45] by few adjustments to deal with MOPs. Similar antibodies are inhibited in VAIS, and a number of new antibodies are added by random sampling to increase the population diversity. Furthermore, an immune dominance clonal multi-objective algorithm (IDCMA) was designed in [35], which adopts Pareto domination relationship to determine the procedures of clonal selection with binary string representation. The improved version of IDCMA is a non-dominated neighbor-based immune algorithm (NNIA) [38], which designs a novel neighbor-based selection technique and population retention strategy to guarantee the population diversity using real-coded representation. A dynamic multi-objective immune algorithm was presented for solving constrained nonlinear MOPs [50], which is realized by simulating the simple interactive metaphors between antibody population and multiple antigens. A novel simplified metaphor of immune response is implemented to obtain multiple excellent feasible solutions so as to explore the whole feasible PF. This work is further extended to the application of greenhouse control [51].

More recently, there are many competitive immune algorithms still presented to further enhance the performance. In [39], a hybrid immune multi-objective optimization algorithm (HIMO) was designed by us, which presents a hybrid mutation operator combining Gaussian and polynomial 
mutations. The performance of HIMO was also enhanced by us [40] with an adaptive mutation operator for local search and a fine-grained selection mechanism for archive update. The adaptive mutation operator is executed according to the crowding-distance values, which promotes to use adaptive steps respectively for crowded and less-crowded individuals. A novel multi-objective immune algorithm was implemented by using a multiple-affinity model [52], which uses six measures for affinity assignment. Based on the selected affinity measures, immune operators such as clonal proliferation, hyper-mutation and immune suppression are executed accordingly, which proliferate the superiors and suppress the inferiors. Afterwards, a novel immune clonal algorithm (NICA) [41] was presented with an improved clonal selection strategy, which can overcome the shortcomings of simple immune algorithm and well handle some complicated MOPs; in [53], a multi-objective immune algorithm with Baldwinian learning (MIAB) was designed, in which a Baldwinian learning strategy is presented to improve the search capability of NNIA [38]. The environment information and the evolving history of the parent solution are exploited to generate a predictive improving direction.

All the above-mentioned immune algorithms treat the multiple objectives as a whole and are aimed at optimizing them simultaneously. However, as the conflicts exist among the objectives, the simultaneous optimization on all the objectives may decline the convergence speed on each objective, which is very evident in the case with many local PFs. For example, some of the above immune algorithms [38-41] cannot solve very well within the limited generations for the test problems characterized with many local PFs, e.g., WFG1, DTLZ1 and DTLZ3 [54-55]. Thus, this paper embeds a single-objective optimization process into traditional immune algorithm, which can accelerate the convergence speed for each objective and resultantly enhance the performance. Compared with the above immune algorithms, the distinct feature of our algorithm is the double modules cooperatively evolved for solving MOPs and its superior performance is also confirmed by the experimental studies described in Section 4.

\section{The Proposed Algorithm}

\subsection{Framework of DMMO}

The framework of DMMO is illustrated in Fig. 1, which is composed by initialization and two evolutionary modules, i.e., module I and module II. In the initialization phase, an initial population $P(t=0)=\left\{a_{1}, a_{2}, \ldots, a_{N}\right\}$ with $N$ solutions is randomly generated, where $t$ indicates the iteration number. The first module selects $m$ ( $m$ is the number of the objectives) subpopulations from the archive population, which prefers the solutions with better values in each objective. These sub-populations are represented by $\operatorname{sub}_{j} p_{j}(j=1,2, \ldots, m)$. In module I, DE crossover and polynomial mutation (PM) are utilized to enhance each objective independently. Module II follows 
the general procedures of immune algorithms, including cloning, recombination, mutation and selection operators. Since module I is aimed at optimizing each objective independently by evolving each sub-population, it will pay more attention to the boundaries of PF and resultantly accelerate the convergence speed of each objective. However, the optimization of single objective in module I may lead most of solutions to exploring the boundaries of PF. Thus, it is necessary to use module II that guarantees the simultaneous optimization of all objectives and maintains the population diversity. The cooperation of double modules can improve both of the convergence speed and the population diversity.

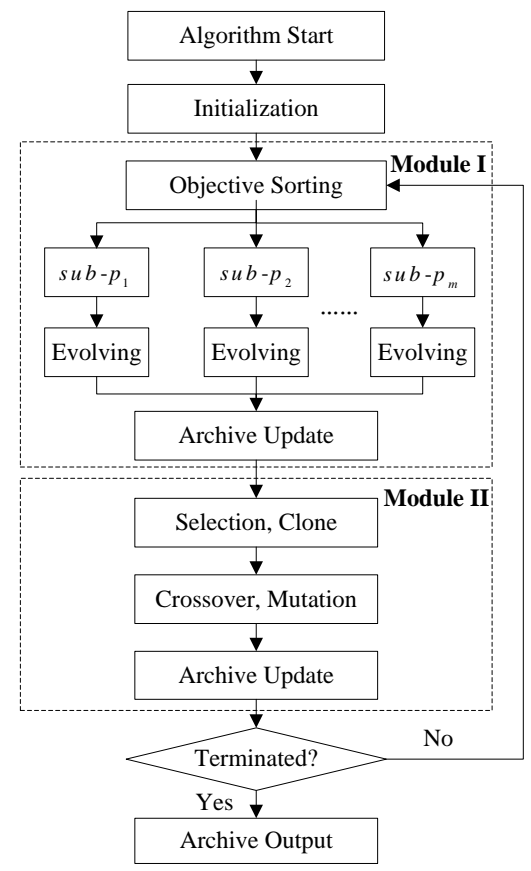

Figure 1. The framework of DMMO

\subsection{Evolutionary Module I}

As indicated before, module I pays more attention to improving the convergence speed of each objective. It guides the search direction focusing on the boundaries of PF, which is achieved by evolving each sub-population with two evolutionary operators independently. After that, some better offspring will be remained that are achieved by an archive update mechanism. In general, the main procedures of module I include sub-population selection, DE and PM operators, and archive update, which are respectively described as follows.

\subsubsection{Sub-population Selection}

Sub-population selection is an important step in module I. Firstly, it needs to obtain the objective matrix by calculating the $j$-th $(j=1,2, \ldots, m)$ objective value of $i$-th $(i=1,2, \ldots, N)$ solution, as represented by $f(P)=\left\{\left[f_{1}(P)\right],\left[f_{2}(P)\right], \ldots,\left[f_{m}(P)\right]\right\}$, where $P$ is the population 
including $N$ individuals. Secondly, ranking selection is performed in each $\left[f_{j}(P)\right](j=1,2, \ldots, m)$ by sorting the objective values in an ascending order. At last, select the first $n a$ solutions for each objective and keep them in each $s u b-p_{j}=\left\{p_{1}, p_{2}, \ldots p_{n a}\right\} \quad(j=1,2, \ldots, m)$, where the value of $n a$ is dependent on the population size $N$ and suggested to be $(10 \% \times N)$ based on the experimental studies. Here, it is pointed out that when the ranking selection is executed, the intersection between two sub-populations is allowed, as it exists the possibility that some individuals are better in multiple objectives. In other words, the individual with better fitness can be selected to build multiple sub-populations.

\subsubsection{Differential Evolution Operator}

Differential evolution is a very powerful crossover operator, which has appeared in many studies and widely applied in numerous practical application areas [27, 56]. In DMMO, DE is adopted as crossover operator in module I. In each subpopualtion $s u b-p_{j}=\left\{p_{1}, p_{2}, \ldots p_{n a}\right\}$ $(j=1,2, \ldots, m)$, na best solutions in the $j$-th objective are neighboring with each other. DE is exactly fit for the solutions with neighboring relationship, since it is good for local search on neighboring solutions [27]. Assume that each solution in $s u b-p_{j}$ is represented by $p_{i}=\left(p_{i}^{1}, p_{i}^{2}, \ldots, p_{i}^{n}\right)$ $(i=1,2, \ldots, n a)$, where $n$ is the dimension of the decision vector. When performing DE on each $p_{i}$, two distinct parents $p_{a}$ and $p_{b}$ are randomly selected from $s u b-p_{j}$, and then a child solution $d_{i}$ is produced as defined in Eq. (2).

$$
\begin{cases}d_{i}=p_{i}+F \times\left(p_{a}-p_{b}\right) & r<C R \\ d_{i}=p_{i} & r \geq C R\end{cases}
$$

where $r$ is a uniformly random number in $[0,1], C R$ is the crossover rate and $F$ is the scaling factor controlling the adjustment range. It is noted that if the new generated child variable is out of the boundary value, it is re-assigned as the corresponding boundary value.

\subsubsection{Polynomial Mutation Operator}

After DE operator, the child solution $d_{i}=\left\{d_{i}^{1}, d_{i}^{2}, \ldots, d_{i}^{n}\right\}$ is obtained and further mutated by PM operator to do local disturbance. The resultant solution $e_{i}=\left(e_{i}^{1}, e_{i}^{2}, \ldots, e_{i}^{n}\right)$ is got by using PM operator [7-8, 38-39], as defined in Eq. (3).

$$
e_{i}^{k}=d_{i}^{k}+\delta \times\left(y u^{k}-y d^{k}\right), k=1,2, \ldots n
$$

where $y u^{k}$ and $y d^{k}$ are respectively the upper and lower bounds of the $k$-th decision variable, and the value of $\delta$ follows a polynomial distribution, as generated by

$$
\delta= \begin{cases}{\left[2 r+(1-2 r) \times \delta_{1}^{\eta+1}\right]^{1 /(\eta+1)}-1,} & \text { if } r<0.5 \\ 1-\left[2(1-r)+2(r-0.5) \times \delta_{2}{ }^{\eta+1}\right]^{1 /(\eta+1)} & \text { if } r \geq 0.5\end{cases}
$$


where $r$ is a uniformly random number in $[0,1]$ and $\eta$ is the distribution index. Generally, a bigger value of $\eta$ will lead to a smaller change of $d_{i}^{k}$. The values of $\delta_{1}$ and $\delta_{2}$ are obtained by

$$
\delta_{1}=\frac{d_{i}^{k}-y d^{k}}{y u^{k}-y d^{k}} \text { and } \delta_{2}=\frac{y u^{k}-d_{i}^{k}}{y u^{k}-y d^{k}}
$$

If the value of $e_{i}^{k}$ is out of the bounds, the corresponding boundary value will be assigned to $e_{i}^{k}$.

\subsubsection{Archive Update}

After the above evolutionary operators are executed, all the new generated offspring are collected into the archive. Once the size of archive is larger than the predefined maximal size $N$, only the best $N$ solutions will be retained in the archive. At first, all the non-dominated solutions are selected by a non-dominated sorting-based method [7]. If the size of non-dominated solutions is still bigger than $N$, a fine-grained selection mechanism designed by us [40] is adopted, in which the most crowded solution will be deleted and the crowding-distance values of its neighbors are updated dynamically. After that, only the solutions with bigger crowding-distance values will be kept in the archive. Once the size of archive is reduced to $N$, module I is switched to module II.

\subsection{Evolutionary Module II}

After the execution of module I, some better solutions in archive will be evolved using module II, which is used to optimize all the objectives and guarantee the population diversity. Based on the procedures of traditional immune algorithm, this module includes three important immune operators, i.e., cloning, crossover and mutation. Proportional cloning operator is used to duplicate the better solutions in archive, where the clones are propagated proportionally with the crowding-distance values. The solutions with larger crowding-distance values will have more clones. After that, the simulated binary crossover (SBX) and PM operators are respectively performed to evolve the clones. At last, all the mutated clones are collected into the archive and the procedure of archive update is activated as introduced in Section 3.2.4.

\subsubsection{Proportional Cloning Operator}

In this module, only a small proportion of better individuals in the archive are selected for cloning. To promote the exploration on the entail PF, the individuals with bigger crowding-distance values are chosen, which are represented by $A=\left\{a_{1}, a_{2}, \ldots, a_{n_{A}}\right\}$, where $n_{A}$ is the number of selected individuals. Assuming that the total clones are equal to $N$, the number of clones $q_{i}$ for each $a_{i}\left(i=1,2, \ldots, n_{A}\right)$ is calculated by Eq. (4).

$$
q_{i}=\left|N \times f i t\left(a_{i}\right) / \sum_{j=1}^{n_{A}} f i t\left(a_{j}\right)\right|
$$

where $f i t\left(a_{i}\right)$ returns the fitness value of $a_{i}$ that is set to be the crowding-distance value [7]. 
Therefore, the individual $a_{i}$ with better $f i t\left(a_{i}\right)$ has a larger value of $q_{i}$, which has more chances to generate duplicated individuals. By this way, the less-crowded solutions have more clones that can benefit the search within the entire search space.

\subsubsection{Recombination and Mutation Operators}

Simulated binary crossover and PM operators are used as the crossover and mutation operators respectively in module II. SBX is one of main recombination operators used in various real-coded multi-objective algorithms [7-8, 38-40], which performs a similar search pattern with the single-point crossover. The child solution is created dependent on the location of the parents. Assume the clone population after the proportional cloning is represented by $C=\left\{c_{1}, c_{2}, \ldots, c_{N}\right\}$. For each individual $c_{i}=\left(c_{i}^{1}, c_{i}^{2}, \ldots, c_{i}^{n}\right) \quad(i=1,2, \ldots, N)$, another parent $a_{j}$ is randomly selected from $A$ (the selected individuals for cloning in Section 3.3.1), where $j$ is a uniformly random integer in $\left[1, n_{A}\right]$. Let $u^{k}$ and $v^{k}(k=1,2, \ldots, n)$ be $\max \left(c_{i}^{k}, a_{j}^{k}\right)$ and $\min \left(c_{i}^{k}, a_{j}^{k}\right)$. Two child decision variables $t_{1}^{k}$ and $t_{2}^{k}$ are obtained by using SBX, as defined in Eqs. (5) and (6) respectively.

$$
\begin{aligned}
& t_{1}^{k}=0.5 \times\left[\left(u^{k}+v^{k}\right)+\beta \times\left(u^{k}-v^{k}\right)\right] \\
& t_{2}^{k}=0.5 \times\left[\left(u^{k}+v^{k}\right)-\beta \times\left(u^{k}-v^{k}\right)\right]
\end{aligned}
$$

where $\beta$ is the dithering amplitude that is got by

$$
\beta= \begin{cases}{[r \times \alpha]^{1 /(\eta+1)}} & \text { if } r \leq 1 / \alpha \\ {[1 /(2-r \times \alpha)]^{1 /(\eta+1)}} & \text { if } r>1 / \alpha\end{cases}
$$

where $r$ is a uniformly random number in $[0,1], \eta$ is a crossover distribution index and $\alpha$ is defined by

$$
\alpha=\left\{\begin{array}{l}
2-\left(\frac{1}{1+2 \times\left(y u^{k}-u^{k}\right) /\left(u^{k}-v^{k}\right)}\right)^{\eta+1} \text { for } t_{1}^{k} \\
2-\left(\frac{1}{1+2 \times\left(v^{k}-y d^{k}\right) /\left(u^{k}-v^{k}\right)}\right)^{\eta+1} \text { for } t_{2}^{k}
\end{array}\right.
$$

where $y u^{k}$ and $y d^{k}$ are respectively the upper and lower bounds of the $k$-th decision variable. Generally, the value of $\beta$ in Eq. (7) becomes closer to 1 with the increase of $\eta$. Thus, the child solutions will have higher probability to be sampled around their parents according to Eqs. (5) and (6). After SBX, the resultant child solution $d_{i}=\left\{d_{i}^{1}, d_{i}^{2}, \ldots, d_{i}^{n}\right\} \quad(i=1,2, \ldots, N)$ is obtained by randomly assigning $d_{i}^{k}$ with $t_{1}^{k}$ or $t_{2}^{k}(k=1,2, \ldots, n)$.

After that, PM operator is further executed on each $d_{i}(i=1,2, \ldots, N)$, which has been introduced in Section 3.2.2. The new generated solutions are then added into the archive. The archive update operation is activated when the size of archive is larger than $N$, which has been described in Section 3.2.3. At last, if the termination condition is not satisfied, module II is turned to module I. 
Otherwise, the non-dominated solutions in the archive are exported as the approximate PF.

\section{Simulations}

\subsection{Benchmark Problems}

In this study, two scalable benchmark problem suites, i.e., WFG [54] and DTLZ [55] problem suites, are selected to assess the performance of DMMO. All the WFG and DTLZ benchmark problems (WFG1-WFG9 and DTLZ1-DTLZ7) are adopted, which are characterized with convexity, concavity, multimodal and non-uniformity. It is noted that the WFG and DTLZ problem suites are respectively scaled to two and three objectives. They are widely used in various MOEAs [7-8, 30-31, 38-41] to evaluate the comprehensive performance. The implementation of all the WFG and DTLZ problems can be found in jMetal [57].

\subsection{Performance Metric}

The goal of MOPs has two important aspects. One is to minimize the distance between the approximate PF and the true PF. The other is to maximize the spread of the approximate PF. In our experiments, two widely used performance metrics, i.e., inverted generational distance (IGD) metric [27] and Hypervolume (HV) metric [14], are adopted to compare the performance of all the algorithms. Both of them can measure the convergence and diversity of the approximate PF simultaneously.

1) Inverted generational distance (IGD) metric [27]: Assuming that the set of approximate PF acquired by an algorithm is $A$, and setting the true PF be $P$, the IGD metric is computed in Eq. (8).

$$
\operatorname{IGD}(P, A)=\frac{\sum_{i=1}^{|P|} d\left(P_{i}, A\right)}{|P|}
$$

where $|P|$ returns the size of $P$ and $d\left(P_{i}, A\right)$ represents the minimum normalized Euclidean distance in the objective space between $P_{i}$ and the solutions in set $A$. Generally speaking, the lower value of IGD metric indicates the better convergence and diversity of the obtained result.

2) Hypervolume (HV) metric [14]: Let $Z^{r}=\left(\mathrm{z}_{1}^{r}, \mathrm{z}_{2}^{r}, \ldots, \mathrm{z}_{m}^{r}\right)^{T}$ be a reference point in the objective space that is dominated by all Pareto-optimal objective vectors, which can be built simply by constructing a vector of worst values in each objective. HV metric measures the volumes of objective space dominated by the solutions in set $A$, which can be calculated by using Eq. (9).

$$
\operatorname{HV}(A)=\operatorname{Vol}\left(U_{x \in A}\left[f_{1}(x), z_{1}^{r}\right] \times\left[f_{2}(x), z_{2}^{r}\right] \ldots \times\left[f_{m}(x), z_{m}^{r}\right]\right)
$$

where the function $\operatorname{Vol}(\cdot)$ indicates the Lebesgue measure. The larger value of the HV metric represents a better quality of $A$ for approaching the true PF. According to the suggestion in [17], the normalized objective function values are used to compute the HV metric and the reference point is 
simply set as $(2,2)$ and $(2,2,2)$ respectively for bi-objective and triple-objective test problems in our experiments.

\subsection{Experimental Settings}

In this section, in order to assess the performance of DMMO in a persuadable way, DMMO is compared with various multi-objective algorithms including two classical MOEAs (NSGA-II and SPEA2) [7, 8], a hypervolume-based MOEA (SMSEMOA) [18], a decomposition-based MOEA (MOEA/D) [27] and a new PSO-based metaheuristic for MOPs (SMPSO) [31]. The implementation of above algorithms can be found in jMetal [57]. Furthermore, DMMO is also compared with two recently proposed multi-objective immune algorithms, i.e., NNIA [38] and MIMO [41]. The source codes of NNIA, MIMO and DMMO are realized by us in jMetal [57]. As all the compared algorithms have shown promising performance in handling various MOPs, the comparisons of DMMO with them can effectively examine its performance.

The parameter settings of these algorithms are shown in TABLE I. For all the algorithms, $N$ is the population and the archive sizes, $p_{c}$ is the crossover probability and $p_{m}$ is the mutation probability. $\eta_{c}$ and $\eta_{m}$ are respectively the distribution indexes of SBX and PM. In MOEA/D, $w_{n}$ defines the size of the neighborhood in the weight coefficients, $\delta$ controls the probability that the parent solutions are chosen from the neighborhood population or the entire population, and $n_{r}$ is the maximal number of the neighbors that can be replaced by offspring solutions. For SMPSO, $C_{1}$ and $C_{2}$ are two control parameters randomly generated from [1.5, 2.5], which adjust the influence of the personal and global best particles to the new particle. For NNIA, MIMO and DMMO, $n_{A}$ is the number of individuals that are selected for cloning. $n a$ is the size of each sub-population in module I of DMMO. For all the test problems, the population size is set to 100 and the maximal number of function evaluations (FEs) is kept at 25000 for all the algorithms. Thus, for NSGA-II, SPEA2, SMSEMOA, SMPSO, MOEA/D, NNIA and MIMO, 250 generations are performed to achieve the maximal FEs as 100 FEs are executed in each generation $(250 \times 100=25000)$. However, regarding to our algorithm DMMO, the generation times depend on the number of objectives and the maximal FEs. In each generation, it will have $(10 \times m)$ FEs in module I and 100 FEs in module II for the test problems, where $m$ is the number of objectives. 30 independent runs are performed for each test problem, in which the mean values and the standard deviations are adopted for comparison. Note that the lowest values of IGD and the largest values of HV are identified with bold font, which indicate the best results obtained in the comparisons. Moreover, the Wilcoxon's rank sum test is performed to examine whether the results obtained by DMMO are different from that obtained by the other algorithms. When the value of Wilcoxon's rank sum test is less than 5\%, it indicates that the obtained results by distinct algorithms are different in statistical sense. 
TABLE I

The parameter settings of all the algorithms

\begin{tabular}{ll}
\hline Algorithm & Parameter settings \\
\hline NSGA-II & $N=100, p_{c}=1.0, p_{m}=1 / n, n_{c}=20, n_{m}=20$ \\
\hline SPEA2 & $N=100, p_{c}=1.0, p_{m}=1 / n, n_{c}=20, n_{m}=20$ \\
\hline SMSEMOA & $N=100, p_{c}=1.0, p_{m}=1 / n, n_{c}=20, n_{m}=20$ \\
\hline MOEA/D & $N=100, C R=1.0, F=0.5, p_{m}=1 / n, n_{m}=20, w_{m}=20, \delta=0.9, n_{r}=2$ \\
\hline SMPSO & $N=100, C_{1} \in[1.5,2.5], C_{2} \in[1.5,2.5], p_{m}=1 / n, n_{m}=20$ \\
\hline NNIA & $N=100, p_{c}=1.0, n_{A}=20, p_{m}=1 / n, n_{c}=20, n_{m}=20$ \\
\hline DIMO & $N=100, p_{c}=1.0, n_{A}=20, p_{m}=1 / n, n_{c}=20, n_{m}=20$ \\
\hline
\end{tabular}

\subsection{Comparisons of DMMO with Several Multi-objective Algorithms}

\subsubsection{Comparison on the WFG Test Problems}

TABLE II gives the IGD results of all the algorithms on the WFG test problems. It is noted that the rank of each algorithm for each problem is obtained by comparing their IGD values and the smaller standard deviations will be preferred in the case with the same IGD values. The results indicate that most of the compared algorithms can well solve WFG2-WFG9. However, for WFG1, only our proposed algorithm DMMO achieves a very close approximate PF as our IGD value is under the level of $10^{-3}$ while other algorithms only get the IGD value on the level of $10^{-1}$. As observed in Table II, DMMO achieves the best results on WFG1-WFG3 and WFG5; SMSEMOA performs best on WFG4, WFG7 and WFG9; SMPSO and NSGA-II respectively get the best values on WFG6 on WFG8. The Wilcoxon's rank sum test shows that DMMO achieves the similar results with NSGA-II on WFG6, with SPEA2 on WFG2 and WFG6, with SMSEMOA on WFG6, with NNIA on WFG8, and with MIMO on WFG4-WFG6, and WFG8. In the last row of TABLE II, the total rank is obtained by summing all the ranks of the compared algorithms in solving each WFG problem. The total rank indicates that DMMO performs best when considering all the WFG problems. Moreover, SPEA2, MIMO, SMSEMOA, NSGA-II, SMPSO, NNIA and MOEA/D, respectively have $2^{\text {nd }}, 3^{\text {rd }}, 4^{\text {th }}, 5^{\text {th }}, 6^{\text {th }}, 7^{\text {th }}$ and $8^{\text {th }}$ ranks. Therefore, the advantages of DMMO on the WFG problems are validated in this experiment. To further illustrate the converging behaviors of all the algorithms, Fig. 2 shows the converging plots of all the algorithms on the WFG test problems, where the IGD values are the averages of 30 runs that are obtained in each 500 FEs. 
TABLE II

The IGD results of all the compared algorithms on the WFG test problems

\begin{tabular}{|c|c|c|c|c|c|c|c|c|c|}
\hline \multicolumn{2}{|c|}{ Problems } & NSGA-II & SPEA2 & MOEA/D & SMPSO & NNIA & MIMO & SMSEMOA & DMMO \\
\hline \multirow{3}{*}{ WFG1 } & Mean & $1.36 \mathrm{E}-1$ & $2.44 \mathrm{E}-1$ & $1.97 \mathrm{E}-1$ & $4.64 \mathrm{E}-1$ & $1.13 \mathrm{E}-1$ & $1.33 \mathrm{E}-1$ & $2.93 \mathrm{E}-1$ & 8.98E-3 \\
\hline & Std & 8.97E-2 & $6.66 \mathrm{E}-2$ & $1.27 \mathrm{E}-1$ & $1.10 \mathrm{E}-2$ & $1.07 \mathrm{E}-1$ & $9.80 \mathrm{E}-2$ & $8.51 \mathrm{E}-2$ & $1.71 \mathrm{E}-2$ \\
\hline & Rank & 4 & 6 & 5 & 8 & 2 & 3 & 7 & 1 \\
\hline \multirow{3}{*}{ WFG2 } & Mean & $4.02 \mathrm{E}-3$ & $3.50 \mathrm{E}-3$ & $9.92 \mathrm{E}-3$ & $3.79 \mathrm{E}-3$ & $4.56 \mathrm{E}-3$ & $3.75 \mathrm{E}-3$ & $3.80 \mathrm{E}-3$ & 3.34E-3 \\
\hline & Std & $5.08 \mathrm{E}-4$ & $6.38 \mathrm{E}-4$ & $7.78 \mathrm{E}-4$ & $2.66 \mathrm{E}-3$ & $5.30 \mathrm{E}-4$ & $6.47 \mathrm{E}-4$ & $5.53 \mathrm{E}-4$ & $5.94 \mathrm{E}-4$ \\
\hline & Rank & 6 & $2 \approx$ & 8 & 4 & 7 & 3 & 5 & 1 \\
\hline \multirow{3}{*}{ WFG3 } & Mean & $4.80 \mathrm{E}-3$ & $4.02 \mathrm{E}-3$ & $4.40 \mathrm{E}-2$ & $4.17 \mathrm{E}-3$ & $4.23 \mathrm{E}-2$ & $4.18 \mathrm{E}-2$ & $4.39 \mathrm{E}-2$ & 3.80E-3 \\
\hline & Std & $1.92 \mathrm{E}-4$ & $1.53 \mathrm{E}-4$ & $8.49 \mathrm{E}-5$ & $1.53 \mathrm{E}-4$ & $2.08 \mathrm{E}-4$ & $8.02 \mathrm{E}-5$ & $9.32 \mathrm{E}-5$ & $1.01 E-4$ \\
\hline & Rank & 4 & 2 & 8 & 3 & 6 & 5 & 7 & 1 \\
\hline \multirow{3}{*}{ WFG4 } & Mean & $5.00 \mathrm{E}-3$ & $4.94 \mathrm{E}-3$ & $6.64 \mathrm{E}-3$ & $1.19 \mathrm{E}-2$ & $5.08 \mathrm{E}-3$ & $4.14 \mathrm{E}-3$ & 3.82E-3 & $4.11 \mathrm{E}-3$ \\
\hline & Std & $2.70 \mathrm{E}-4$ & $1.74 \mathrm{E}-4$ & $2.06 \mathrm{E}-4$ & $1.13 \mathrm{E}-3$ & $2.99 \mathrm{E}-3$ & $9.83 \mathrm{E}-5$ & 7.67E-5 & $1.01 \mathrm{E}-4$ \\
\hline & Rank & 5 & 4 & 7 & 8 & 6 & $3 \approx$ & 1 & 2 \\
\hline \multirow{3}{*}{ WFG5 } & Mean & $2.74 \mathrm{E}-2$ & $2.73 \mathrm{E}-2$ & $2.80 \mathrm{E}-2$ & $2.74 \mathrm{E}-2$ & $2.78 \mathrm{E}-2$ & $2.73 \mathrm{E}-2$ & $2.74 \mathrm{E}-2$ & 2.73E-2 \\
\hline & Std & $1.43 \mathrm{E}-4$ & $1.03 \mathrm{E}-3$ & $2.06 \mathrm{E}-5$ & $4.58 \mathrm{E}-5$ & $1.88 \mathrm{E}-4$ & $4.96 \mathrm{E}-4$ & $2.56 \mathrm{E}-5$ & 5.68E-5 \\
\hline & Rank & 6 & 3 & 8 & 5 & 7 & $2 \approx$ & 4 & 1 \\
\hline \multirow{3}{*}{ WFG6 } & Mean & $1.26 \mathrm{E}-2$ & $7.84 \mathrm{E}-3$ & $2.39 \mathrm{E}-2$ & $4.22 \mathrm{E}-3$ & $1.85 \mathrm{E}-2$ & $1.66 \mathrm{E}-2$ & $1.28 \mathrm{E}-2$ & $1.21 \mathrm{E}-2$ \\
\hline & Std & $1.01 \mathrm{E}-2$ & $5.74 \mathrm{E}-3$ & $1.99 \mathrm{E}-2$ & 1.19E-4 & $1.17 \mathrm{E}-2$ & $1.15 \mathrm{E}-2$ & $1.15 \mathrm{E}-4$ & $1.04 \mathrm{E}-2$ \\
\hline & Rank & $4 \approx$ & $2 \approx$ & 8 & 1 & 7 & $6 \approx$ & $5 \approx$ & 3 \\
\hline \multirow{3}{*}{ WFG7 } & Mean & $4.98 \mathrm{E}-3$ & $4.20 \mathrm{E}-3$ & $5.70 \mathrm{E}-3$ & $3.93 \mathrm{E}-3$ & $4.92 \mathrm{E}-3$ & $3.94 \mathrm{E}-3$ & 3.34E-3 & $3.89 \mathrm{E}-3$ \\
\hline & Std & $3.45 \mathrm{E}-4$ & $1.71 \mathrm{E}-4$ & $2.52 \mathrm{E}-5$ & $7.72 \mathrm{E}-5$ & $3.54 \mathrm{E}-4$ & $9.77 \mathrm{E}-5$ & 9.61E-5 & $7.51 \mathrm{E}-5$ \\
\hline & Rank & 7 & 5 & 8 & 3 & 6 & 4 & 1 & 2 \\
\hline \multirow{3}{*}{ WFG8 } & Mean & 6.42E-2 & $6.78 \mathrm{E}-2$ & $7.36 \mathrm{E}-2$ & 8.32E-2 & $7.34 \mathrm{E}-2$ & $6.78 \mathrm{E}-2$ & $9.16 \mathrm{E}-2$ & $6.84 \mathrm{E}-2$ \\
\hline & Std & $1.98 \mathrm{E}-2$ & $1.35 \mathrm{E}-2$ & $2.97 \mathrm{E}-2$ & $6.58 \mathrm{E}-3$ & $1.49 \mathrm{E}-4$ & $1.92 \mathrm{E}-2$ & $3.51 \mathrm{E}-3$ & $1.93 \mathrm{E}-2$ \\
\hline & Rank & 1 & 2 & 6 & 7 & $5 \approx$ & $3 \approx$ & 8 & 4 \\
\hline \multirow{3}{*}{ WFG9 } & Mean & $6.03 \mathrm{E}-3$ & $5.32 \mathrm{E}-3$ & $6.50 \mathrm{E}-3$ & $6.50 \mathrm{E}-3$ & $6.62 \mathrm{E}-3$ & $5.57 \mathrm{E}-3$ & 4.32E-3 & $4.94 \mathrm{E}-3$ \\
\hline & Std & $6.59 \mathrm{E}-4$ & $6.39 \mathrm{E}-4$ & $1.02 \mathrm{E}-3$ & $2.50 \mathrm{E}-4$ & $1.43 \mathrm{E}-3$ & $1.33 \mathrm{E}-3$ & $6.36 \mathrm{E}-4$ & 7.69E-4 \\
\hline & Rank & 5 & 3 & 7 & 6 & 8 & 4 & 1 & 2 \\
\hline \multicolumn{2}{|c|}{ Total Rank } & 42 & 29 & 65 & 45 & 54 & 33 & 39 & 17 \\
\hline
\end{tabular}

' $\approx$ ' indicates the results obtained by the algorithm are similar to the results obtained by DMMO at a 5\% significant level by the Wilcoxon's rank sum test.

TABLE III lists the HV results of all the compared algorithms on the WFG test problems. Similar results are observed on TABLE III. DMMO achieves the best values on WFG1-WFG3 and WFG5 and SMPSO performs best on WFG6 and WFG8; SMSEMOA is best on WFG4 and WFG9, and MIMO gets the best result on WFG7. The Wilcoxon's rank sum test shows that DMMO achieves the similar results with NSGA-II on WFG2, WFG6 and WFG8, with SPEA2 on WFG2, WFG6 and WFG9, with MOEA/D on WFG6, WFG8 and WFG9, with SMPSO on WFG2, with NNIA on WFG8, with MIMO on WFG4 and WFG7-WFG9, and with SMSEMOA on WFG2 and WFG6. The total rank in last row of TABLE III also indicates that DMMO performs best when considering all the WFG test problems. Therefore, the advantages of DMMO on the WFG test problems are confirmed by using the HV metric. 

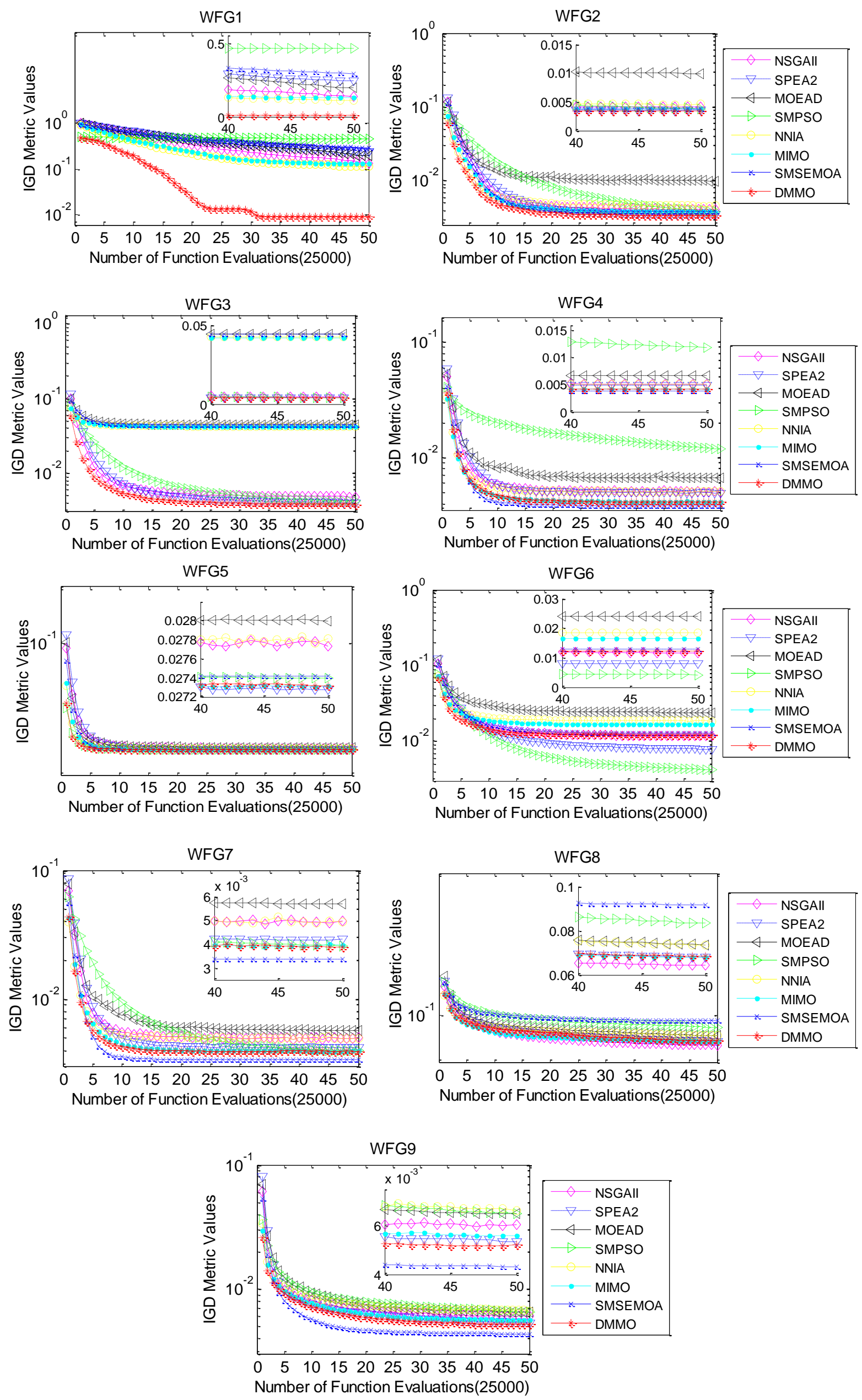

Figure 2. The converging plots of DMMO on the WFG problems. 
TABLE III

The HV results of all the compared algorithms on the WFG test problems

\begin{tabular}{|c|c|c|c|c|c|c|c|c|c|}
\hline \multicolumn{2}{|c|}{ Problems } & NSGA-II & SPEA2 & MOEA/D & SMPSO & NNIA & MIMO & SMSEMOA & DMMO \\
\hline \multirow{3}{*}{ WFG1 } & Mean & 3.2423 & 2.9141 & 3.1191 & 2.3600 & 3.3125 & 3.2685 & 2.7717 & 3.5843 \\
\hline & Std & $2.94 \mathrm{E}-1$ & $2.19 \mathrm{E}-1$ & $2.89 \mathrm{E}-1$ & $1.91 \mathrm{E}-2$ & $3.05 \mathrm{E}-1$ & $2.73 \mathrm{E}-1$ & $2.72 \mathrm{E}-1$ & $1.21 \mathrm{E}-1$ \\
\hline & Rank & 4 & 6 & 5 & 8 & 2 & 3 & 7 & 1 \\
\hline \multirow{3}{*}{ WFG2 } & Mean & 3.4509 & 3.4491 & 3.4446 & 3.4560 & 3.4265 & 3.4148 & 3.4407 & 3.4594 \\
\hline & Std & $9.23 \mathrm{E}-2$ & $9.02 \mathrm{E}-2$ & $8.83 \mathrm{E}-2$ & $7.56 \mathrm{E}-2$ & $7.45 \mathrm{E}-2$ & 7.39E-2 & $8.68 \mathrm{E}-2$ & 9.16E-2 \\
\hline & Rank & $3 \approx$ & $4 \approx$ & 5 & $2 \approx$ & 7 & 8 & $6 \approx$ & 1 \\
\hline \multirow{3}{*}{ WFG3 } & Mean & 3.4907 & 3.4906 & 3.1062 & 3.4911 & 3.4908 & 3.1068 & 3.1076 & 3.4937 \\
\hline & Std & $1.02 \mathrm{E}-3$ & $1.60 \mathrm{E}-3$ & $8.84 \mathrm{E}-4$ & $7.82 \mathrm{E}-4$ & $8.30 \mathrm{E}-4$ & $1.13 \mathrm{E}-3$ & $7.14 \mathrm{E}-4$ & 6.89E-4 \\
\hline & Rank & 4 & 5 & 8 & 2 & 3 & 7 & 6 & 1 \\
\hline \multirow{3}{*}{ WFG4 } & Mean & 3.2172 & 3.2175 & 3.2175 & 3.1901 & 3.2174 & 3.2187 & 3.2190 & 3.2188 \\
\hline & Std & $4.81 \mathrm{E}-4$ & $6.63 \mathrm{E}-4$ & $2.66 \mathrm{E}-4$ & $6.60 \mathrm{E}-3$ & $2.69 \mathrm{E}-4$ & $2.49 \mathrm{E}-4$ & $6.02 E-3$ & $2.30 \mathrm{E}-4$ \\
\hline & Rank & 7 & 5 & 4 & 8 & 6 & $3 \approx$ & 1 & 2 \\
\hline \multirow{3}{*}{ WFG5 } & Mean & 3.1788 & 3.1672 & 3.1621 & 3.1651 & 3.1886 & 3.1868 & 3.1690 & 3.1896 \\
\hline & Std & $1.26 \mathrm{E}-2$ & $8.50 \mathrm{E}-3$ & $1.04 \mathrm{E}-3$ & $7.90 \mathrm{E}-3$ & $9.17 \mathrm{E}-3$ & $9.05 \mathrm{E}-3$ & $9.69 \mathrm{E}-3$ & $4.75 \mathrm{E}-3$ \\
\hline & Rank & 4 & 6 & 8 & 7 & 2 & 3 & 5 & 1 \\
\hline \multirow{3}{*}{ WFG6 } & Mean & 3.1806 & 3.1805 & 3.1792 & 3.2072 & 3.1447 & 3.1484 & 3.1805 & 3.1769 \\
\hline & Std & $2.08 \mathrm{E}-2$ & $2.90 \mathrm{E}-2$ & $2.91 \mathrm{E}-2$ & 8.44E-4 & $5.92 \mathrm{E}-2$ & $4.08 \mathrm{E}-1$ & $2.96 \mathrm{E}-2$ & $3.58 \mathrm{E}-2$ \\
\hline & Rank & $2 \approx$ & $3 \approx$ & $5 \approx$ & 1 & 8 & 7 & $4 \approx$ & 6 \\
\hline \multirow{3}{*}{ WFG7 } & Mean & 3.2082 & 3.2057 & 3.2055 & 3.2084 & 3.2092 & 3.2104 & 3.2077 & 3.2104 \\
\hline & Std & $9.48 \mathrm{E}-4$ & $2.10 \mathrm{E}-3$ & $2.58 \mathrm{E}-3$ & $5.18 \mathrm{E}-4$ & $3.45 \mathrm{E}-4$ & 1.13E-4 & $1.91 \mathrm{E}-3$ & $1.34 \mathrm{E}-4$ \\
\hline & Rank & 5 & 7 & 8 & 4 & 3 & $1 \approx$ & 6 & 2 \\
\hline \multirow{3}{*}{ WFG8 } & Mean & 2.8498 & 2.8186 & 2.8666 & 2.8774 & 2.8718 & 2.8617 & 2.8202 & 2.8460 \\
\hline & Std & $6.58 \mathrm{E}-2$ & $3.88 \mathrm{E}-2$ & $8.84 \mathrm{E}-2$ & 2.99E-2 & 7.05E-2 & $9.11 \mathrm{E}-2$ & $5.80 \mathrm{E}-2$ & $5.47 \mathrm{E}-2$ \\
\hline & Rank & $5 \approx$ & 8 & $3 \approx$ & 1 & $2 \approx$ & $4 \approx$ & 7 & 6 \\
\hline \multirow{3}{*}{ WFG9 } & Mean & 3.2367 & 3.2380 & 3.2371 & 3.2350 & 3.2364 & 3.2375 & 3.2401 & 3.2378 \\
\hline & Std & $2.09 \mathrm{E}-3$ & $3.60 \mathrm{E}-3$ & $2.33 \mathrm{E}-3$ & $5.68 \mathrm{E}-4$ & $2.72 \mathrm{E}-3$ & $3.77 \mathrm{E}-3$ & $1.39 \mathrm{E}-3$ & $3.11 \mathrm{E}-3$ \\
\hline & Rank & 6 & $2 \approx$ & $5 \approx$ & 8 & 7 & $4 \approx$ & 1 & 3 \\
\hline \multicolumn{2}{|c|}{ Total Rank } & 40 & 46 & 51 & 41 & 40 & 40 & 43 & 23 \\
\hline
\end{tabular}

' $\approx$ ' indicates the results obtained by the algorithm are similar to the results obtained by DMMO at a 5\% significant level by the Wilcoxon's rank sum test.

\subsubsection{Comparison on the DTLZ Test Problems}

In TABLE IV, the IGD results of all the algorithms on the DTLZ test problems are illustrated. It is noted that most of the compared algorithms cannot well solve DTLZ3 as it exists many local PFs, while SMPSO, DMMO and MOEA/D performs comparatively better in DTLZ3 as their IGD values are under the level of $10^{-1}$. For DTLZ6, DMMO achieves the much better result than that obtained by the other algorithms. As observed in TABLE IV, DMMO obtains the best result on DTLZ6 and performs secondly on DTLZ1-DTLZ2 and DTLZ4-DTLZ5, while SMPSO performs best on DTLZ3-DTLZ5; NSGA-II, SPEA2 and SMSEMOA respectively achieve the best result on DTLZ7, DTLZ2 and DTLZ1. Moreover, the Wilcoxon's rank sum test demonstrates that DMMO has the similar results with NSGA-II on DTLZ1 and DTLZ4, with SPEA2 on DTLZ1, with SMSEMOA on DTLZ4, with MOEA/D on DTLZ3, with SMPSO on DTLZ4, with NNIA on DTLZ4 and DTLZ7, and with MIMO on DTLZ4, DTLZ5 and DTLZ7. According to the total rank in TABLE IV, SMPSO performs slightly better than DMMO and gets the first rank, while DMMO achieves the second rank 
on all the DTLZ test problems. Besides that, SPEA2, MIMO and NNIA respectively rank at $3^{\text {rd }}, 4^{\text {th }}$ and $5^{\text {th }}$. NSGA-II and SMSEMOA obtain the same rank at $6^{\text {th }}$, while MOEA/D performs worst on these test problems.

TABLE IV

The IGD results of all the compared algorithms on the DTLZ test problems

\begin{tabular}{|c|c|c|c|c|c|c|c|c|c|}
\hline \multicolumn{2}{|c|}{$\begin{array}{l}\text { Algorithms } \\
\text { Problems }\end{array}$} & NSGA-II & SPEA2 & MOEA/D & SMPSO & NNIA & MIMO & SMSEMOA & DMMO \\
\hline \multirow{3}{*}{ DTLZ1 } & Mean & $2.08 \mathrm{E}-1$ & $7.35 \mathrm{E}-2$ & $6.55 \mathrm{E}-2$ & $5.81 \mathrm{E}-2$ & $1.65 \mathrm{E}-1$ & $1.14 \mathrm{E}-1$ & 3.98E-2 & $5.49 \mathrm{E}-2$ \\
\hline & Std & $2.63 \mathrm{E}-1$ & $1.02 \mathrm{E}-1$ & $1.27 \mathrm{E}-3$ & $3.58 \mathrm{E}-3$ & $3.31 \mathrm{E}-1$ & $1.70 \mathrm{E}-1$ & 4.57E-4 & $1.74 \mathrm{E}-2$ \\
\hline & Rank & $8 \approx$ & $5 \approx$ & 4 & 3 & 7 & 6 & 1 & 2 \\
\hline \multirow{3}{*}{ DTLZ2 } & Mean & $6.84 \mathrm{E}-2$ & $5.46 \mathrm{E}-2$ & $7.31 \mathrm{E}-2$ & $7.16 \mathrm{E}-2$ & $6.90 \mathrm{E}-2$ & $6.92 \mathrm{E}-2$ & $7.39 \mathrm{E}-2$ & $6.61 \mathrm{E}-2$ \\
\hline & Std & $3.09 \mathrm{E}-3$ & 8.98E-3 & $3.88 \mathrm{E}-4$ & $3.24 \mathrm{E}-3$ & $2.42 \mathrm{E}-3$ & $3.40 \mathrm{E}-3$ & $1.05 \mathrm{E}-3$ & $2.92 \mathrm{E}-3$ \\
\hline & Rank & 3 & 1 & 7 & 6 & 4 & 5 & 8 & 2 \\
\hline \multirow{3}{*}{ DTLZ3 } & Mean & $7.00 \mathrm{E}+0$ & $5.88 \mathrm{E}+0$ & $4.37 \mathrm{E}-1$ & 1.49E-1 & $2.96 \mathrm{E}+0$ & $3.02 \mathrm{E}+0$ & $2.98 \mathrm{E}+0$ & $5.31 \mathrm{E}-1$ \\
\hline & Std & $3.62 \mathrm{E}+0$ & $3.04 \mathrm{E}+0$ & $6.44 \mathrm{E}-1$ & 1.11E-1 & $1.92 \mathrm{E}+0$ & $2.45 \mathrm{E}+0$ & $2.21 \mathrm{E}+0$ & $5.80 \mathrm{E}-1$ \\
\hline & Rank & 8 & 7 & $2 \approx$ & 1 & 4 & 6 & 5 & 3 \\
\hline \multirow{3}{*}{ DTLZ4 } & Mean & $6.43 \mathrm{E}-2$ & $8.89 \mathrm{E}-2$ & $1.83 \mathrm{E}-1$ & 6.06E-2 & $8.63 \mathrm{E}-2$ & $9.87 \mathrm{E}-2$ & $1.27 \mathrm{E}-1$ & $6.20 \mathrm{E}-2$ \\
\hline & Std & $6.14 \mathrm{E}-3$ & $8.19 \mathrm{E}-2$ & $1.31 \mathrm{E}-1$ & $1.44 \mathrm{E}-2$ & $9.24 \mathrm{E}-2$ & $1.11 \mathrm{E}-1$ & $1.10 \mathrm{E}-1$ & $5.31 \mathrm{E}-3$ \\
\hline & Rank & $3 \approx$ & 5 & 8 & $1 \approx$ & $4 \approx$ & $6 \approx$ & $7 \approx$ & 2 \\
\hline \multirow{3}{*}{ DTLZ5 } & Mean & $6.46 \mathrm{E}-3$ & $5.55 \mathrm{E}-3$ & $1.87 \mathrm{E}-2$ & 5.00E-3 & $6.69 \mathrm{E}-3$ & $5.18 \mathrm{E}-3$ & $5.92 \mathrm{E}-3$ & $5.12 \mathrm{E}-3$ \\
\hline & Std & $4.32 \mathrm{E}-4$ & $1.77 \mathrm{E}-4$ & $1.08 \mathrm{E}-5$ & $1.46 \mathrm{E}-4$ & $3.51 \mathrm{E}-4$ & $1.57 \mathrm{E}-4$ & $1.14 \mathrm{E}-4$ & $1.37 \mathrm{E}-4$ \\
\hline & Rank & 6 & 4 & 8 & 1 & 7 & $3 \approx$ & 5 & 2 \\
\hline \multirow{3}{*}{ DTLZ6 } & Mean & $1.15 \mathrm{E}+0$ & $1.14 \mathrm{E}+0$ & $5.45 \mathrm{E}-2$ & $1.39 \mathrm{E}-2$ & $5.14 \mathrm{E}-1$ & $5.33 \mathrm{E}-1$ & $3.12 \mathrm{E}-1$ & 5.09E-3 \\
\hline & Std & $1.11 \mathrm{E}-1$ & $8.66 \mathrm{E}-2$ & $2.36 \mathrm{E}-2$ & $4.40 \mathrm{E}-2$ & $1.01 \mathrm{E}-1$ & $9.45 \mathrm{E}-2$ & $6.18 \mathrm{E}-2$ & $1.47 \mathrm{E}-4$ \\
\hline & Rank & 8 & 7 & 3 & 2 & 5 & 6 & 4 & 1 \\
\hline \multirow{3}{*}{ DTLZ7 } & Mean & $5.06 \mathrm{E}-2$ & $6.08 \mathrm{E}-2$ & $1.19 \mathrm{E}-1$ & $5.94 \mathrm{E}-2$ & $6.12 \mathrm{E}-2$ & $6.05 \mathrm{E}-2$ & $1.05 \mathrm{E}-1$ & $7.70 \mathrm{E}-2$ \\
\hline & Std & $2.47 \mathrm{E}-3$ & $6.24 \mathrm{E}-2$ & $7.53 \mathrm{E}-4$ & $3.92 \mathrm{E}-3$ & $4.34 \mathrm{E}-2$ & 4.30E-2 & $4.10 \mathrm{E}-2$ & $7.25 \mathrm{E}-2$ \\
\hline & Rank & 1 & 4 & 8 & 2 & $5 \approx$ & $3 \approx$ & 7 & 6 \\
\hline \multicolumn{2}{|c|}{ Total Rank } & 37 & 33 & 40 & 16 & 36 & 35 & 37 & 18 \\
\hline
\end{tabular}

' $\approx$ ' indicates the results obtained by the algorithm are similar to the results obtained by DMMO at a $5 \%$ significant level by the Wilcoxon's rank sum test.

Moreover, the HV results of all the compared algorithms on the DTLZ test problems are listed in TABLE V. DMMO gets the best results on DTLZ6, and performs secondly on DTLZ1 and DTLZ5. SMSEMOA is best on DTLZ1, DTLZ2 and DTLZ5, while NSGA-II, SPEA2 and SMPSO respectively obtain the best results on DTLZ4, DTLZ7 and DTLZ3. Besides that, the Wilcoxon's rank sum test indicates DMMO has the similar results with SPEA2 on DTLZ1 and DTLZ4, with MOEA/D on DTLZ3, with SMPSO on DTLZ3 and DTLZ5, with NNIA on DTLZ4 and DTLZ7, with MIMO on DTLZ4, DTLZ5 and DTLZ7, and with SMSEMOA on DTLZ1. The total rank in last row of TABLE V illustrates that DMMO and SMSEMOA respectively get the first and second ranks when concerning all the DTLZ test problems. Moreover, SMPSO and MOEA/D respectively achieve the $3^{\text {th }}$ and $4^{\text {th }}$ ranks; SPEA2 and MIMO perform similarly and both get the $5^{\text {th }}$ rank; at last, NSGA-II and NNIA obtain the $7^{\text {th }}$ and $8^{\text {th }}$ ranks respectively. 
TABLE V

The HV results of all the compared algorithms on the DTLZ test problems

\begin{tabular}{|c|c|c|c|c|c|c|c|c|c|}
\hline Problems & rithms & NSGA-II & SPEA2 & MOEA/D & SMPSO & NNIA & MIMO & SMSEMOA & DMMO \\
\hline \multirow{3}{*}{ DTLZ1 } & Mean & 7.5786 & 7.7342 & 7.7414 & 7.7319 & 7.3627 & 7.3060 & 7.7862 & 7.7661 \\
\hline & Std & $5.18 \mathrm{E}-1$ & $1.28 \mathrm{E}-1$ & $3.75 \mathrm{E}-3$ & $8.20 \mathrm{E}-3$ & $5.89 \mathrm{E}-1$ & $7.42 \mathrm{E}-1$ & 2.04E-3 & $4.10 \mathrm{E}-3$ \\
\hline & Rank & 6 & $4 \approx$ & 3 & 5 & 7 & 8 & 1 & 2 \\
\hline \multirow{3}{*}{ DTLZ2 } & Mean & 7.3520 & 7.3930 & 7.3780 & 7.3459 & 7.3708 & 7.3714 & 7.4222 & 7.3849 \\
\hline & Std & $1.73 \mathrm{E}-2$ & $1.09 \mathrm{E}-2$ & $1.04 \mathrm{E}-3$ & $8.20 \mathrm{E}-3$ & $1.05 \mathrm{E}-2$ & $1.04 \mathrm{E}-2$ & 1.07E-4 & $4.42 \mathrm{E}-3$ \\
\hline & Rank & 7 & 2 & 4 & 8 & 6 & 5 & 1 & 3 \\
\hline \multirow{3}{*}{ DTLZ3 } & Mean & 0.1934 & 0.0000 & 5.2771 & 6.8594 & 0.8500 & 0.7056 & 1.0105 & 4.6132 \\
\hline & Std & $9.94 \mathrm{E}-1$ & $0.00 \mathrm{E}+0$ & $2.48 \mathrm{E}+0$ & 8.16E-2 & $2.44 \mathrm{E}+0$ & $1.48 \mathrm{E}+0$ & $2.87 \mathrm{E}+0$ & $2.91 \mathrm{E}+0$ \\
\hline & Rank & 7 & 8 & $2 \approx$ & $1 \approx$ & 5 & 6 & 4 & 3 \\
\hline \multirow{3}{*}{ DTLZ4 } & Mean & 7.3671 & 6.8076 & 6.8095 & 7.3551 & 7.1531 & 7.0409 & 7.0121 & 7.2676 \\
\hline & Std & $7.65 \mathrm{E}-3$ & 8.82E-1 & $1.02 \mathrm{E}+0$ & $1.04 \mathrm{E}-2$ & $8.43 \mathrm{E}-1$ & $1.01 \mathrm{E}+0$ & $1.21 \mathrm{E}+0$ & $6.07 \mathrm{E}-1$ \\
\hline & Rank & 1 & $8 \approx$ & 7 & 2 & $4 \approx$ & $5 \approx$ & 6 & 3 \\
\hline \multirow{3}{*}{ DTLZ5 } & Mean & 5.5118 & 5.5135 & 5.5123 & 5.5151 & 5.5112 & 5.5153 & 5.5212 & 5.5155 \\
\hline & Std & $7.16 \mathrm{E}-4$ & $5.62 \mathrm{E}-4$ & $3.56 \mathrm{E}-3$ & $3.71 \mathrm{E}-4$ & 8.72E-4 & $2.80 \mathrm{E}-4$ & 3.78E-5 & $2.17 \mathrm{E}-4$ \\
\hline & Rank & 7 & 5 & 6 & $4 \approx$ & 8 & $3 \approx$ & 1 & 2 \\
\hline \multirow{3}{*}{ DTLZ6 } & Mean & 0.9915 & 0.8307 & 5.4042 & 5.3667 & 3.7280 & 3.6289 & 5.3521 & 5.5195 \\
\hline & Std & $4.59 \mathrm{E}-1$ & 8.24E-2 & $4.62 \mathrm{E}-2$ & $8.21 \mathrm{E}-2$ & $9.11 \mathrm{E}-1$ & $5.84 \mathrm{E}-1$ & 7.33E-2 & 8.35E-5 \\
\hline & Rank & 7 & 8 & 2 & 3 & 5 & 6 & 4 & 1 \\
\hline \multirow{3}{*}{ DTLZ7 } & Mean & 6.5177 & 6.5325 & 6.4933 & 6.4957 & 6.4486 & 6.4961 & 6.4615 & 6.4641 \\
\hline & Std & $2.14 \mathrm{E}-2$ & 4.54E-2 & $1.37 \mathrm{E}-1$ & $2.67 \mathrm{E}-2$ & 2.34E-1 & $1.96 \mathrm{E}-1$ & $4.42 \mathrm{E}-1$ & $1.92 \mathrm{E}-1$ \\
\hline & Rank & 2 & 1 & 5 & 4 & $8 \approx$ & $3 \approx$ & 7 & 6 \\
\hline \multicolumn{2}{|c|}{ Total Rank } & 37 & 36 & 29 & 27 & 43 & 36 & 24 & 20 \\
\hline
\end{tabular}

' $\approx$ ' indicates the results obtained by the algorithm are similar to the results obtained by DMMO at a $5 \%$ level by the Wilcoxon's rank sum test.

TABLE VI

The total ranks of all the compared algorithms on the IGD and HV metrics for all the test problems

\begin{tabular}{|c|c|c|c|c|c|c|c|c|}
\hline Problems & NSGA-II & SPEA2 & MOEA/D & SMPSO & NNIA & MIMO & SMSEMOA & DMMO \\
\hline WFG1-9 (IGD) & 42 & 29 & 65 & 45 & 54 & 33 & 39 & 17 \\
\hline WFG1-9 (HV) & 40 & 46 & 51 & 41 & 40 & 40 & 43 & 23 \\
\hline DTLZ1-7 (IGD) & 37 & 33 & 40 & 16 & 36 & 35 & 37 & 18 \\
\hline DTLZ1-7 (HV) & 37 & 36 & 29 & 27 & 43 & 36 & 24 & 20 \\
\hline Total ranks & 156 & 144 & 185 & 129 & 173 & 144 & 143 & 78 \\
\hline
\end{tabular}

In TABLE VI, the total ranks of all the compared algorithms on the IGD and HV metrics for solving all the WFG and DTLZ test problems are collected. From the last row of TABLE VI, it is obvious that DMMO has the best performance in solving all the test problems when considering both of the IGD and HV metrics. Moreover, to graphically show the convergence and spread of the final non-dominated solutions, Figs. 3 and 4 respectively give the plots of 50\% attainment surfaces obtained by DMMO in solving all the WFG and DTLZ test problems, where the true PFs are marked with red line or surface and the $50 \%$ attainment surfaces are identified with black arrows. It is noted 
the 50\% attainment surfaces is obtained using the approach reported in [58-59]. These plots illustrate that the $50 \%$ attainment surfaces found by DMMO are very close to the true PFs and distributed uniformly on the true PFs except WFG8.
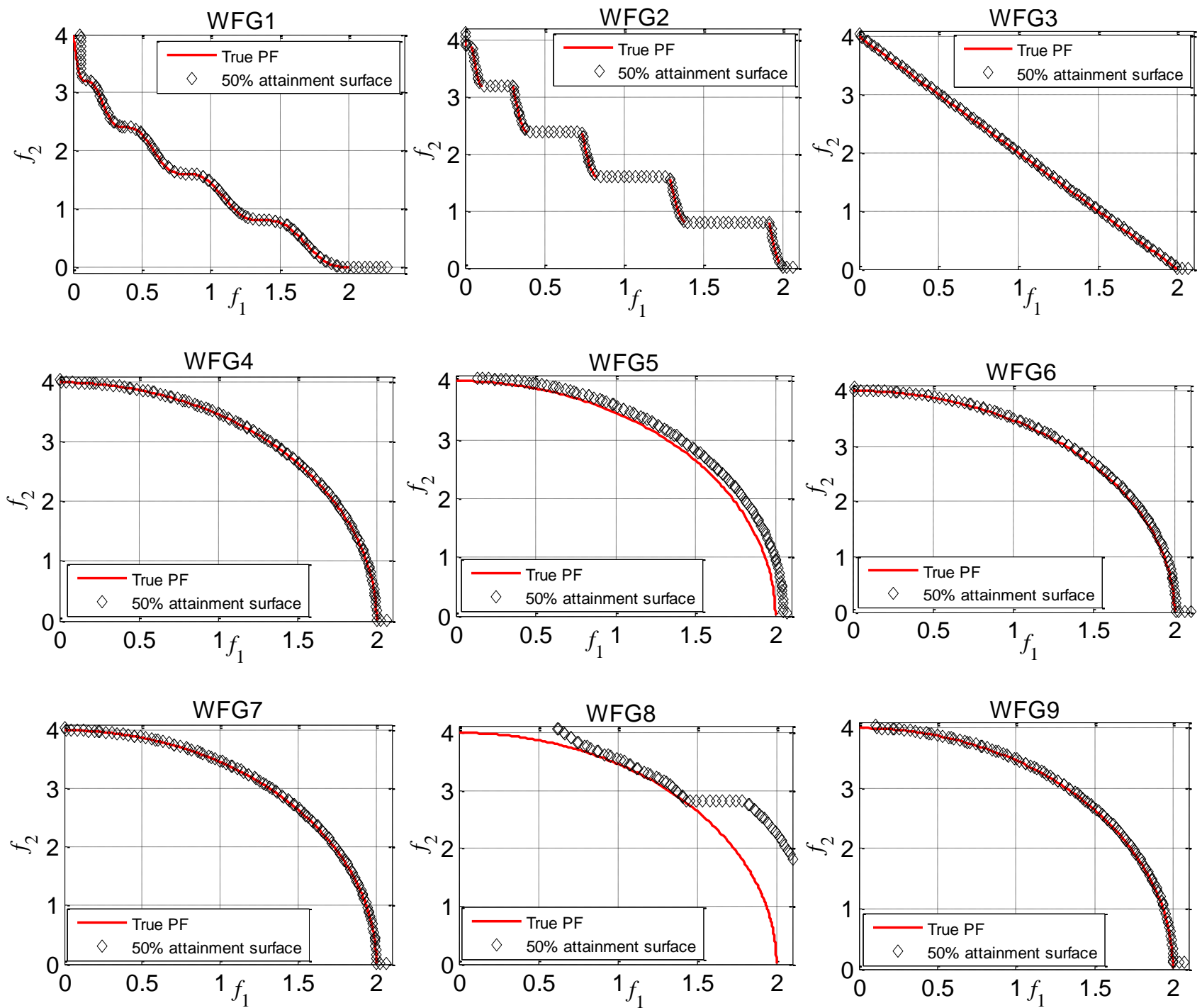

Figure 3. The final non-dominated solutions of DMMO in the WFG problems 

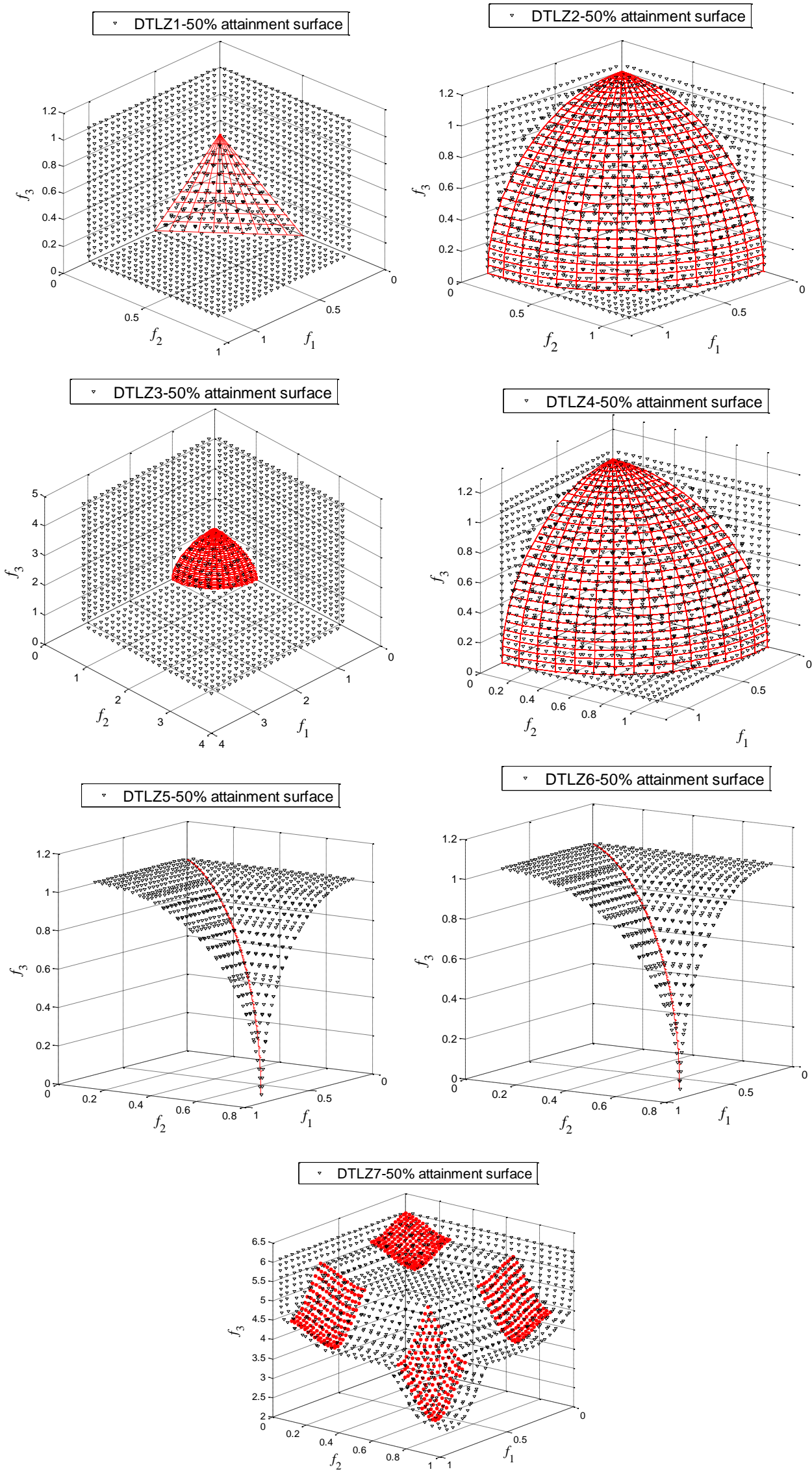

Figure 4. The final non-dominated solutions of DMMO in the DTLZ problems 


\subsection{Advantages of Double Modules and Related Operators}

In the above subsections, it is experimentally validated that DMMO has superior performance when compared with NSGA-II, SPEA2, SMSEMOA, MOEA/D, SMPSO, NNIA and MIMO. In order to investigate the advantages of double modules and DE operator in module I, two experimental studies are conducted. One is to compare DMMO with DMMO-1, in which DE operator in module I is replaced by SBX operator. The other is to compare DMMO with DMMO-2, where module I in DMMO is removed. TABLE VII demonstrates the IGD results of DMMO-1, DMMO-2 and DMMO on all the WFG and DTLZ test problems.

TABLE VII

The IGD results of DMMO-1, DMMO-2 and DMMO on all the WFG and DTLZ test problems

\begin{tabular}{|c|c|c|c|c|c|c|c|c|c|}
\hline \multicolumn{2}{|c|}{ Problems } & \multirow{2}{*}{$\frac{\text { DMMO-1 }}{1.00 \mathrm{E}-1}$} & \multirow{2}{*}{$\frac{\text { DMMO-2 }}{1.60 \mathrm{E}-1}$} & \multirow{2}{*}{$\frac{\text { DMMO }}{\text { 8.98E-3 }}$} & \multicolumn{2}{|c|}{ Problems } & \multirow{2}{*}{$\frac{\text { DMMO-1 }}{5.23 \mathrm{E}-3}$} & \multirow{2}{*}{$\frac{\mathrm{DMMO}-2}{5.51 \mathrm{E}-3}$} & \multirow{2}{*}{$\frac{\text { DMMO }}{\mathbf{4 . 9 4 E - 3}}$} \\
\hline \multirow{3}{*}{ WFG1 } & Mean & & & & \multirow{3}{*}{ WFG9 } & Mean & & & \\
\hline & Std & 8.67E-2 & $1.23 \mathrm{E}-1$ & $1.71 \mathrm{E}-2$ & & Std & $1.60 \mathrm{E}-3$ & $1.83 \mathrm{E}-3$ & 7.69E-4 \\
\hline & Rank & 2 & 3 & 1 & & Rank & $2 \approx$ & 3 & 1 \\
\hline \multirow{3}{*}{ WFG2 } & Mean & $3.91 \mathrm{E}-3$ & $4.07 \mathrm{E}-3$ & 3.34E-3 & \multirow{3}{*}{ DTLZ1 } & Mean & $1.71 \mathrm{E}-1$ & $2.36 \mathrm{E}-1$ & 5.49E-2 \\
\hline & Std & $5.82 \mathrm{E}-4$ & $6.21 \mathrm{E}-4$ & 5.94E-4 & & Std & $2.12 \mathrm{E}-1$ & $3.08 \mathrm{E}-1$ & $1.74 \mathrm{E}-2$ \\
\hline & Rank & 2 & 3 & 1 & & Rank & 2 & 3 & 1 \\
\hline \multirow{3}{*}{ WFG3 } & Mean & $3.94 \mathrm{E}-3$ & $4.15 \mathrm{E}-3$ & 3.80E-3 & \multirow{3}{*}{ DTLZ2 } & Mean & $6.95 \mathrm{E}-2$ & $6.93 \mathrm{E}-2$ & $6.61 \mathrm{E}-2$ \\
\hline & Std & $3.09 \mathrm{E}-4$ & $4.30 \mathrm{E}-4$ & 1.01E-4 & & Std & $3.56 \mathrm{E}-3$ & $3.81 \mathrm{E}-3$ & 2.92E-3 \\
\hline & Rank & 2 & 3 & 1 & & Rank & 3 & 2 & 1 \\
\hline \multirow{3}{*}{ WFG4 } & Mean & $4.15 \mathrm{E}-3$ & $4.15 \mathrm{E}-3$ & $4.11 \mathrm{E}-3$ & \multirow{3}{*}{ DTLZ3 } & Mean & $1.94 \mathrm{E}+0$ & $2.71 \mathrm{E}+0$ & 5.31E-1 \\
\hline & Std & $7.04 \mathrm{E}-5$ & $6.92 \mathrm{E}-5$ & 1.01E-4 & & Std & $1.56 \mathrm{E}+0$ & $2.48 \mathrm{E}+0$ & $5.80 \mathrm{E}-1$ \\
\hline & Rank & 3 & $2 \approx$ & 1 & & Rank & 2 & 3 & 1 \\
\hline \multirow{3}{*}{ WFG5 } & Mean & $2.76 \mathrm{E}-2$ & $2.73 E-2$ & $2.73 \mathrm{E}-2$ & \multirow{3}{*}{ DTLZ4 } & Mean & $1.60 \mathrm{E}-1$ & $1.26 \mathrm{E}-1$ & $6.20 \mathrm{E}-2$ \\
\hline & Std & $4.36 \mathrm{E}-4$ & $2.57 \mathrm{E}-5$ & $5.68 \mathrm{E}-5$ & & Std & $1.62 \mathrm{E}-1$ & $1.36 \mathrm{E}-1$ & 5.31E-3 \\
\hline & Rank & $3 \approx$ & $1 \approx$ & 2 & & Rank & 3 & 2 & 1 \\
\hline \multirow{3}{*}{ WFG6 } & Mean & $1.79 \mathrm{E}-2$ & $1.56 \mathrm{E}-2$ & $1.21 \mathrm{E}-2$ & \multirow{3}{*}{ DTLZ5 } & Mean & $5.18 \mathrm{E}-3$ & $5.14 \mathrm{E}-3$ & 5.12E-3 \\
\hline & Std & $1.33 \mathrm{E}-2$ & $1.10 \mathrm{E}-2$ & 1.04E-2 & & Std & $1.57 \mathrm{E}-4$ & $1.68 \mathrm{E}-4$ & 1.37E-4 \\
\hline & Rank & 3 & $2 \approx$ & 1 & & Rank & $3 \approx$ & $2 \approx$ & 1 \\
\hline \multirow{3}{*}{ WFG7 } & Mean & $3.88 \mathrm{E}-3$ & $3.95 \mathrm{E}-3$ & $3.89 \mathrm{E}-3$ & \multirow{3}{*}{ DTLZ6 } & Mean & $1.19 \mathrm{E}-1$ & $5.20 \mathrm{E}-1$ & 5.09E-3 \\
\hline & Std & 9.39E-5 & $1.09 \mathrm{E}-4$ & $7.51 \mathrm{E}-5$ & & Std & $8.29 \mathrm{E}-2$ & $1.00 \mathrm{E}-1$ & $1.47 \mathrm{E}-4$ \\
\hline & Rank & $1 \approx$ & 3 & 2 & & Rank & 2 & 3 & 1 \\
\hline \multirow{3}{*}{ WFG8 } & Mean & $7.32 \mathrm{E}-2$ & $7.23 \mathrm{E}-2$ & $6.84 \mathrm{E}-2$ & \multirow{3}{*}{ DTLZ7 } & Mean & $1.08 \mathrm{E}-1$ & 8.39E-2 & $7.70 \mathrm{E}-2$ \\
\hline & Std & $1.38 \mathrm{E}-2$ & $1.55 \mathrm{E}-2$ & 1.93E-2 & & Std & $1.16 \mathrm{E}-1$ & 8.14E-2 & $7.25 \mathrm{E}-2$ \\
\hline & Rank & $3 \approx$ & $2 \approx$ & 1 & & Rank & $3 \approx$ & $1 \approx$ & 2 \\
\hline \multicolumn{2}{|c|}{ Total Rank } & 39 & 39 & 18 & & & & & \\
\hline
\end{tabular}

' $\approx$ ' indicates the results obtained by the algorithm are similar to the results obtained by DMMO at a $5 \%$ level by the Wilcoxon's rank sum test.

It is found that the final results of DMMO-1 and DMMO-2 are very similar and the best results are almost found by DMMO. For WFG1, DTLZ1, DTLZ3, DTLZ4 and DTLZ6, the performance of DMMO is much better than that obtained by DMMO-1 and DMMO-2, which in turn illustrates that module I and its relevant DE operator are all very important for DMMO. When concerning the WFG test problems, DMMO all performs better than DMMO-1 and DMMO-2 except WFG5 and WFG7. All the algorithms have similar performance on WFG5. For WFG7, DMMO performs similarly with 
DMMO-1 and better than DMMO-2. Regarding to the DTLZ test problems, DMMO obtains the best results on DTLZ1-DTZL6, and performs similarly with DMMO-1 and DMMO-2 on DTLZ7. The total rank in last row of TABLE VII clearly shows that DMMO has the obvious advantages when compared with DMMO-1 and DMMO-2. Thus, these experiments not only confirm the effectiveness of double modules in DMMO, but also validate the superiority of DE operator adopted in module I.

\section{More Discussions of Module I}

As indicated by the above experimental studies, it is pointed out that the single-objective enhancement in module I is very important for DMMO. The reason is that, for some test problems that always have many local optimums, it is too difficult to find the boundary solutions of the approximate PF. A promising solution is to use some mechanisms to explore the boundary solutions, which is aimed at reducing the probability of premature convergence and ensuring the population diversity. When DE operator is performed in module I of DMMO, it is effective to get better solutions for each objective as the two parents are selected from the same sub-population with better fitness values on the corresponding objective. For MOPs, it means that the Pareto-optimal solutions on the boundaries of PF can be explored quickly. Here, Fig. 5 shows the approximate PF of WFG1, where the solutions that are acquired by module I are marked with the red color stars, while the others obtained by module II are identified with the black color points. It can be observed clearly from Fig. 5 that most of the solutions around the boundaries are produced by module I and other solutions in the central regions are all explored by module II. Thus, it is reasonable to conclude that module I is good for finding the solutions around the boundaries while module II is beneficial for maintaining the population diversity. The cooperation of module I and module II is in favor of improving the comprehensive performance of DMMO.

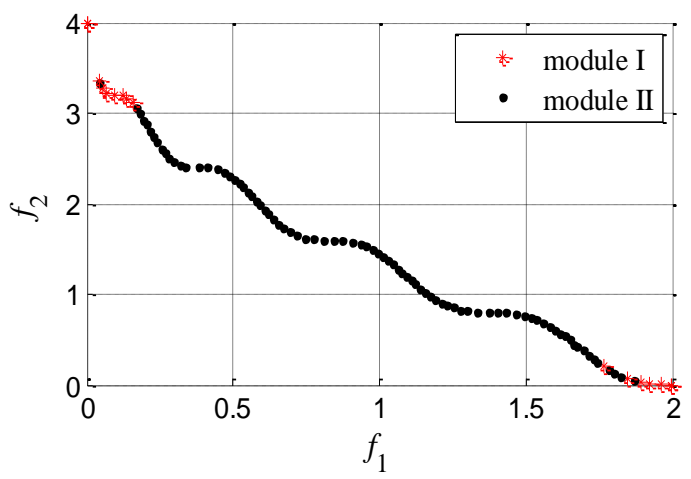

Figure 5. The approximate PF of WFG1

Moreover, in order to further validate the effectiveness of our idea, we embed module I into other bio-inspired algorithms, e.g., NSGA-II and SMPSO. TABLE VIII gives the IGD results obtained by NSGA-II, NSGA-R, SMPSO, and SMPSO-R in solving all the WFG and DTLZ test problems, where NSGA-R and SMPSO-R are respectively the revised NSGA-II and SMPSO 
with module I presented in this paper. It is noted the Wilcoxon's rank sum test is also run to examine whether the results obtained by the revised algorithms are different from that obtained by the original algorithms, and the similar results are marked with underlines. When comparing the performance between NSGA-II and NSGA-R, the better result on each problem is identified with bold font. As observed from TABLE VIII, NSGA-R performs better than or similar with NSGA-II on 12 out of 16 test problems. Especially for WFG1, DTLZ1, DTZL6, the embedment of module I can greatly improve the performance of NSGA-II. Regarding to the comparison of SMPSO and SMPSO-R, the better result on each problem is also identified with boldface. As indicated by TABLE VIII, SMPSO-R achieves the better results than SMPSO on 11 out of 16 test problems and performs similarly with SMPSO on the other test problems. This is to say, the integration of module I into SMPSO can generally enhance its optimization performance, especially for DTLZ6. These experiments further confirm the advantages of our idea.

TABLE VIII

The IGD results of NSGA-II, NSGA-R, SMPSO and SMPSO-R on all the WFG and DTLZ test problems

\begin{tabular}{|c|c|c|c|c|c|c|c|c|c|c|c|}
\hline Problems & & SGA-II & SUA- & עה & SMPSO-R & Problems & & NSG & NS & 50 & SM \\
\hline \multirow{2}{*}{ WFG1 } & ean & 1 & E-3 & 1 & 5E-1 & \multirow{2}{*}{ WFG9 } & Mean & & & -3 & $6.31 E-3$ \\
\hline & Std & $8.97 \mathrm{E}-2$ & E-4 & $E-2$ & 3.11E-1 & & Std & & & E-4 & 0E-4 \\
\hline \multirow{2}{*}{ WFG2 } & Mean & $4.03 \mathrm{~B}$ & & & E-3 & \multirow{2}{*}{ DTLZ1 } & Mean & & & & E-2 \\
\hline & Std & $5.08 \mathrm{E}-4$ & 4.43E-4 & $2.66 \mathrm{E}-3$ & $2.47 \mathrm{E}-4$ & & Std & & -3 & -3 & E-3 \\
\hline \multirow{2}{*}{ WFG3 } & Mean & $4.80 \mathrm{E}-3$ & & -3 & E-3 & \multirow{2}{*}{ DTLZ2 } & Mean & 6. & E-2 & $E-2$ & E-2 \\
\hline & Std & 1.92E-4 & E-4 & E-4 & 7.11E-5 & & Std & & & -3 & E-3 \\
\hline \multirow{2}{*}{ WFG4 } & Iean & 5.0 & 3 & -2 & $\underline{1.14 \mathrm{E}-2}$ & \multirow{2}{*}{ DTLZ3 } & lean & & & $E-1$ & \\
\hline & Std & $2.70 \mathrm{E}-4$ & $3.34 \mathrm{E}-4$ & $\mathrm{E}-3$ & 8.64E-4 & & Std & $3.62 \mathrm{E}+0$ & $\mathrm{E}+\mathbf{0}$ & & E-2 \\
\hline \multirow{2}{*}{ WFG5 } & Mean & $2.74 \mathrm{E}-2$ & 2.73E-2 & $\underline{2.74 \mathrm{E}-2}$ & $\underline{2.74 \mathrm{E}-2}$ & \multirow{2}{*}{ DTLZ4 } & Mean & $\underline{6.43 \mathrm{E}-2}$ & $\underline{6.30 \mathrm{E}-2}$ & $\underline{6.06 \mathrm{E}-2}$ & $6.62 \mathrm{E}-2$ \\
\hline & Std & $1.43 \mathrm{E}-4$ & 2.06E-4 & 4.58E-5 & $\underline{1.11 \mathrm{E}-4}$ & & Std & 6. & E-3 & E-2 & E-3 \\
\hline \multirow{2}{*}{ WFG6 } & Mean & $1.26 \mathrm{E}-2$ & 8.54E-3 & & 3.99E-3 & \multirow{2}{*}{ DTLZ5 } & Mean & & & $\underline{5.00 \mathrm{E}-3}$ & $2 \mathrm{E}-3$ \\
\hline & Std & & & & 1.02E-4 & & Std & & & & E-4 \\
\hline \multirow{2}{*}{ WFG7 } & Mean & & & $3.93 \mathrm{E}-3$ & E-3 & \multirow{2}{*}{ DTLZ6 } & Mean & $\mathrm{E}+0$ & & E-2 & E-3 \\
\hline & Std & 3.4 & 5 & -5 & E-5 & & Std & E-1 & -4 & $\mathrm{E}-2$ & E-4 \\
\hline \multirow{2}{*}{ WFG8 } & Mean & $6.42 \mathrm{E}-2$ & $\underline{5.32 \mathrm{E}-2}$ & $8.32 \mathrm{E}-2$ & 8.11E-2 & \multirow{2}{*}{ DTLZ7 } & Mean & $5.06 \mathrm{E}-2$ & $5.40 \mathrm{E}-2$ & $\underline{6.03 \mathrm{E}-2}$ & 5.82E-2 \\
\hline & Std & $\underline{1.98 \mathrm{E}-2}$ & $\underline{2.44 \mathrm{E}-2}$ & $6.58 \mathrm{E}-3$ & $6.96 \mathrm{E}-3$ & & Std & $2.47 \mathrm{E}-3$ & $2.78 \mathrm{E}-3$ & $\underline{3.92 \mathrm{E}-3}$ & $\underline{5.57 \mathrm{E}-3}$ \\
\hline
\end{tabular}

\section{Conclusions}

In this paper, a novel immune algorithm with double modules is presented for solving MOPs, which is distinctly different from the traditional immune algorithms. Two modules are designed jointly in DMMO to enhance the comprehensive performance. Module I is aimed at improving each objective independently and resultantly guides the search direction focusing on the boundaries of PF, which can accelerate the convergence speed of each objective. DE and PM operators are performed serially in this module. On the other hand, the purpose of module II is to enhance the population diversity, which guarantees the simultaneous optimization of all the objectives. Therefore, the cooperation of two modules can achieve the enhancement on both of the convergence speed and the 
population diversity, which are justified by the experimental studies. When compared with various MOEAs and two recently proposed immune algorithms, including NSGA-II, SPEA2, SMSEMOA, MOEA/D, SMPSO, NNIA and MIMO, the experimental results show that DMMO performs better and can effectively converge to the approximate PF. Moreover, it is experimentally validated that the proposed module I can be successfully applied to other bio-inspired heuristic algorithms (e.g., NSGA-II and SMPSO) and further enhances their performance. Our future study will further enhance the performance of DMMO, and extend it for solving many objective (more than three objectives) optimization problems and practical engineering problems.

\section{Acknowledgements}

This work was supported by the National Natural Science Foundation of China under Grant 61402291 and 61170283, National High-Technology Research and Development Program ("863" Program) of China under Grant 2013AA01A212, Ministry of Education in the New Century Excellent Talents Support Program under Grant NCET-12-0649, Foundation for Distinguished Young Talents in Higher Education of Guangdong under Grant 2014KQNCX129, Natural Science Foundation of Guangdong Province under Grant S2013040011789 and Shenzhen Technology Plan under Grant JCYJ20130401095947219 and JCYJ20140418095735608. The authors are grateful to both the editor and anonymous reviewers for their constructive comments, which greatly improve the presentation of this paper.

\section{References}

[1] R.L. Daniels, R.J. Chambers. Multiobjective flowshop scheduling. Naval Research Logistics 1990; 37(6): 981-995.

[2] Y. Yuan, H. Xu. Multiobjective flexible job shop scheduling using memetic algorithms. IEEE Transactions on Automation Science and Engineering 2015; 12(1) 336-353.

[3] D.A. Savic, G.A. Walters. Genetic algorithms for the least-cost design of water distribution networks. Journal of Water Resources Planning and Management 1997; 123(2): 67-77.

[4] S. Bureerat, K. Sriworamas. Simultaneous topology and sizing optimization of a water distribution network using a hybrid multiobjective evolutionary algorithm. Applied Soft Computing 2013; 13(8): 3693-3702.

[5] J. Lu, D. Ireland, A. Lewis. Multi-objective optimization in high frequency electro magnetic-an effective technique for Smart Mobile Terminal Antenna (SMTA) design. IEEE Transactions on Evolutionary Computation 2009; 45(3):1072-1075.

[6] W. Kong, T. Chai, S. Yang, J. Ding. A hybrid evolutionary multiobjective optimization strategy for the dynamic power supply problem in magnesia grain manufacturing. Applied Soft Computing 2013; 13(5): 2960-2969. 
[7] K. Deb, A. Pratap, S. Agarwal, T. Meyarivan. A fast and elitist multi-objective genetic algorithm: NSGA-II. IEEE Transactions on Evolutionary Computation 2002; 6(2):182-197.

[8] E. Zitzler, M. Laumanns, L. Thiele. SPEA2: improving the strength Pareto evolutionary algorithm. Technical report 103, Zurich, Switzerland: Computer Engineering and Networks Laboratory (TIK), Swiss Federal Institute of Technology (ETH), 2001.

[9] E. Zitzler. Evolutionary Algorithms for Multiobjective Optimization: Methods and Applications. PhD thesis, ETH Zurich, Switzerland, 1999.

[10]R.E. Steuer. Multiple Criteria Optimization: Theory, Computation, and Application. New York: Wiley, 1986.

[11]J.L. Cohon. Multiobjective Programming and Planning. New York: Academic Press, 1978.

[12]J.D. Schaffer. Multiple objective optimization with vector evaluated genetic algorithms. Proceedings of the first international conference on genetic algorithms, 1985, pp. 93-100.

[13]N. Srinivas, K. Deb. Multi-objective optimization using non-dominated sorting in genetic algorithms. Evolutionary Computation 1994; 2(3): 221-248.

[14]E. Zitzler, L. Thiele. Multi-objective evolutionary algorithms: A comparative case study and the strength Pareto approach. IEEE Transactions on Evolutionary Computation 1999; 3(4): 257-271.

[15]J.D. Knowles, D.W. Corne. Approximating the non-dominated front using the Pareto archived evolution strategy. Evolutionary Computation 2000; 8(2):149-172.

[16]D.W. Corne, J.D. Knowles, M.J. Oates. The Pareto-envelope based selection algorithm for multi-objective optimization. Parallel Problem Solving from Nature-PPSN VI, Lecture Notes in Computer Science, 2000, pp. 839-848.

[17]E. Zitzler, S. Künzli. Indicator-based selection in multiobjective search. Parallel Problem Solving from Nature-PPSN VIII, Lecture Notes in Computer Science 2004; 3242: 832-842.

[18]N. Beume, B. Naujoks and M. Emmerich. SMS-EMOA: Multiobjective selection based on dominated hypervolume. European Journal of Operational Research 2007; 181(3): 1653-1669.

[19]J. Knowles. ParEGO: a hybrid algorithm with on-line landscape approximation for expensive multiobjective optimization problems. IEEE Transactions on Evolutionary Computation 2006; 10(1): 50-66.

[20]C. Igel, N. Hansen, S. Roth. Covariance matrix adaptation for multi-objective optimization. Evolutionary Computation 2007; 15(1): 1-28.

[21]Z.W. Yu, H.S. Wong, D.W. Wang, M. Wei. Neighborhood Knowledge-Based Evolutionary Algorithm for Multiobjective Optimization Problems. IEEE Transactions on Evolutionary Computation 2011; 15(6): 812-831.

[22]C.K Chow, S.Y. Yuen. A Multiobjective Evolutionary Algorithm That Diversifies Population by Its Density. IEEE Transactions on Evolutionary Computation 2012; 16(2): 149-172.

[23]H. Kim, M.-S. Liou. Adaptive directional local search strategy for hybrid multiobjective 
optimization. Applied Soft Computing 2014; 19: 290-311.

[24]H.R. Cheshmehgaz, M.I. Desa, A. Wibowo. An effective model of multiple multi-objective evolutionary algorithms with the assistance of regional multi-objective evolution algorithms: VIPMOEAs. Applied Soft Computing 2013; 13(5): 2863-2895.

[25]J.H. Kim, J.H. Han, Y.H. Kim, S.H. Choi, and E.S. Kim. Preference-based solution selection algorithm for evolutionary multi-objective optimization. IEEE Transactions on Evolutionary Computation 2012; 16 (1): 20-34.

[26]S.F. Adra, T.J. Dodd, I.A. Griffin, P.J. Fleming. Convergence acceleration operator for multi-objective optimization. IEEE Transactions on Evolutionary Computation 2009; 13(4): $825-847$.

[27]H. Li, Q.F. Zhang. Multiobjective optimization problems with complicated Pareto sets, MOEA/D and NSGA-II. IEEE Transactions on Evolution Computation 2009; 13(2): 284-302.

[28]K. Sindhya, K. Miettinen, and K. Deb. A Hybrid Framework for Evolutionary Multi-Objective Optimization. IEEE Transactions on Evolutionary Computation 2013; 17(4): 495-511.

[29]L.C. Jiao, J.J. Luo, R.H. Shang and F. Liu. A modified objective function method with feasible-guiding strategy to solve constrained multi-objective optimization problems. Applied Soft Computing 2014; 14: 363-380.

[30]Z.H. Zhan, J.J. Li, J.N. Cao, J. Zhang, H.S. Chung, Y.H. Shi. Multiple Populations for Multiple Objectives: A Coevolutionary Technique for Solving Multiobjective Optimization Problems. IEEE Transactions on Cybernetics 2013; 42(2):445-463.

[31]A.J. Nebro, J.J. Durillo, J. Garcia-Nieto, C.A. Coello Coello, F. Luna, and E. Alba. SMPSO: A new pso-based metaheuristic for multi-objective optimization. Proceedings of IEEE Symposium on Computational Intelligence in Multi-criteria decision-making, 2009, pp. 66-73.

[32]M. Dorigo, C. Blum. Ant colony optimization theory: A survey. Theoretical Computer Science 2005; 344(2-3): 243-278.

[33]J.G. Yang, Y.B. Zhuang. An improved ant colony optimization algorithm for solving a complex combinatorial optimization problem. Applied Soft Computing 2010; 10(2):653-660.

[34]C.A. Coello Coello, C.N. Cruz. An approach to solve multiobjective optimization problems based on an artificial immune system. Proceedings of First International Conference on Artificial Immune Systems, 2002, pp. 212-221.

[35]L.C. Jiao, M.G. Gong, R.H. Shang, H.F. Du, B. Lu. Clonal selection with immune dominance and energy based multiobjective optimization. Evolutionary Multi-Criterion Optimization, Lecture Notes in Computer Science 2005, 3410: 474-489.

[36]E. Hart, J. Timmis. Application areas of AIS: The past, the present and the future. Applied Soft Computing 2008; 8(1): 191-201.

[37]D. Dasgupta, S. Yu, F. Nino. Recent Advances in Artificial Immune Systems: Models and 
Applications. Applied Soft Computing 2011; 11(2): 1574-1587.

[38]M.G. Gong, L.C. Jiao, H.F. Du, L.F. Bo. Multi-objective immune algorithm with nondominated neighbor-based selection. Evolutionary Computation 2008; 16(2):225-255.

[39]J.Y. Chen, Q.Z. Lin, Z. Ji. A hybrid immune multiobjective optimization algorithm. European Journal of Operational Research 2010; 204(2):294-302.

[40]Q.Z. Lin, J.Y. Chen. A novel mirco-population immune multiobjective optimization algorithm. Computers and Operators Research 2013; 40(6):1590-1601.

[41]R.H. Shang, L.C. Jiao, F. Liu, W.P. Ma. A Novel Immune Clonal Algorithm for MO Problems. IEEE Transactions on Evolutionary Computation 2012; 16(1):35-50.

[42]L. Huang, I.H. Suh, A. Abraham. Dynamic multi-objective optimization based on membrane computing for control of time-varying unstable plants. Information Sciences 2011; 181: 2370-2391.

[43]L. Huang, L. Sun, N. Wang, X. Jin. Multiobjective optimization of simulated moving bed by a kind of tissue P system. Chinese Journal of Chemical Engineering 2007; 15: 683-690.

[44]P.A.N. Bosman, D. Thierens. The balance between proximity and diversity in multiobjective evolutionary algorithms. IEEE Transactions on Evolution Computation 2003, 7(2,): 174-188.

[45]L.N. De Castro, J. Timmis. An artificial immune network for multimodal function optimization. Proceedings of the 2002 Congress on Evolutionary Computation, 2002, pp.699-704.

[46]L.N. De Castro, F.J. Von Zuben. Learning and optimization using the clonal selection principle. IEEE Transactions on Evolutionary Computation 2002; 6(3):239-251.

[47]T. Fukuda, K. Mori, M. Tsukiyama. Immune networks using genetic algorithm for adaptive production scheduling. Proceedings of 15th IFAC World Congress, 1993, volume 3, pp. 57-60.

[48]F. Freschi, M. Repetto. Multi-objective optimization by a modified artificial immune system algorithm. Artificial Immune Systems, Lecture Notes in Computer Science 2005; 3627: 248-261.

[49]F. Freschi, M. Repetto. VIS: an artificial immune network for multi-objective optimization. Engineering Optimization 2006; 38(8): 975-996.

[50]Z.H. Zhang. Immune optimization algorithm for constrained nonlinear multiobjective optimization problems. Applied Soft Computing 2007; 7(3):840-857.

[51]Z.H. Zhang. Multiobjective optimization immune algorithm in dynamic environments and its application to greenhouse control. Applied Soft Computing 2008; 8(2):959-971.

[52]Z.H. Hu. A multiobjective immune algorithm based on a multiple-affinity model. European Journal of Operational Research 2010; 202(1): 60-72.

[53]Y.T. Qi, F. Liu, M.Y. Liu, M.G. Gong, L.C. Jiao. Multi-objective immune algorithm with Baldwinian learning. Applied Soft Computing 2012; 12(8):2654-2674.

[54]S. Huband, L. Barone, L. While, P. Hingston. A scalable multi-objective test problem toolkit. 
Evolutionary Multi-Criterion Optimization. Lecture Notes in Computer Science 2005; 3410: 280-295.

[55]K. Deb, L. Thiele, M. Laumanns, E. Zitzler. Scalable test problems for evolutionary multi-objective optimization. Evolutionary Multiobjective Optimization, Advanced Information and Knowledge Processing 2005; pp. 105-145.

[56]S. Das, P.N. Suganthan. Differential Evolution: A Survey of the State-of-the-Art. IEEE Transactions on Evolutionary Computation 2011; 15(1): 4-31.

[57]J.J. Durillo, A.J. Nebro. jMetal: a Java Framework for Multi-Objective Optimization. Advances in Engineering Software 2011; 42(10): 760-771.

[58]J. Knowles. A summary-attainment-surface plotting method for visualizing the performance of stochastic multiobjective optimizers. Proceedings of 5th International Conference on Intelligent Systems Design and Applications, 2005, pp. 552-557.

[59]C.M. Fonseca and P.J. Fleming. On the performance assessment and comparison of stochastic multiobjective optimizers. Parallel Problem Solving from Nature - PPSN IV, Lecture Notes in Computer Science 1996; 1141: 584-593. 